\title{
Development of a Dual-Pump CARS System for Measurements in a Supersonic Combusting Free Jet
}

\author{
Gaetano Magnotti ${ }^{1}$ and Andrew D. Cutler ${ }^{2}$ \\ The George Washington University, Newport News, VA 23602, USA \\ Paul Danehy ${ }^{3}$ \\ NASA Langley Research Center, Advanced Sensing and Optical Measurements Branch, 1200 Langley Boulevard, \\ Hampton, Virginia 23681, USA
}

\begin{abstract}
This work describes the development of a dual-pump CARS system for simultaneous measurements of temperature and absolute mole fraction of $\mathrm{N}_{2}, \mathrm{O}_{2}$ and $\mathrm{H}_{2}$ in a laboratory scale supersonic combusting free jet. Changes to the experimental set-up and the data analysis to improve the quality of the measurements in this turbulent, high-temperature reacting flow are described. The accuracy and precision of the instrument have been determined using data collected in a Hencken burner flame. For temperature above $800 \mathrm{~K}$, errors in absolute mole fraction are within $1.5,0.5$, and $1 \%$ of the total composition for $\mathrm{N}_{2}$, $\mathrm{O}_{2}$ and $\mathrm{H}_{2}$, respectively. Estimated standard deviations based on 500 single shots are between 10 and $65 \mathrm{~K}$ for the temperature, between 0.5 and $1.7 \%$ of the total composition for $\mathrm{O}_{2}$, and between 1.5 and $3.4 \%$ for $\mathrm{N}_{2}$. The standard deviation of $\mathrm{H}_{2}$ is $\sim 10 \%$ of the average measured mole fraction. Results obtained in the jet with and without combustion are illustrated, and the capabilities and limitations of the dual-pump CARS instrument discussed.
\end{abstract}

\section{Introduction}

$\mathrm{T}$ EMPORALLY and spatially resolved measurements in supersonic combusting flow are needed to provide computational fluid-dynamics (CFD) modelers with experimental data to validate existing turbulent models and develop new ones. Quantitative measurements in scramjet engines are particularly challenging because of the harsh testing environment. Coherent Anti-Stokes Raman Spectroscopy (CARS) is a non-intrusive, instantaneous and spatially resolved, laser-based measurement technique that has been implemented by several researchers to measure temperature and species concentration in scramjet combustors. ${ }^{1-3}$ In CARS, three laser beams, conventionally named pump, probe and Stokes, are focused and crossed at their focal point, where a fourth laser-like beam is generated containing the Raman spectrum of the probed species. Laser frequencies are chosen so that the pump-Stokes frequency difference corresponds to the Raman shift associated with a molecular rotational-vibrational transition. In broadband CARS the Stokes laser is spectrally broad so several transitions are probed simultaneously. In dual-pump (DP) CARS, originally developed by Lucht, ${ }^{4}$ and improved by O'Byrne, ${ }^{5}$ the pump and probe laser beams have different wavelengths, and their role interchanges, so that two different regions of the Raman shift are probed simultaneously. Using this approach it is possible to probe the $\mathrm{O}_{2}$ and $\mathrm{N}_{2}$ Q-branch manifolds as well as three or more pure rotational $\mathrm{H}_{2}$ lines. The technique provides instantaneous and spatially-resolved measurements of temperature, and absolute mole fractions of $\mathrm{N}_{2}, \mathrm{O}_{2}$ and $\mathrm{H}_{2}$ in $\mathrm{H}_{2}$-air flames. At NASA Langley Research Center DPCARS was successfully used to measure temperature, $\mathrm{N}_{2}, \mathrm{O}_{2}$ and $\mathrm{H}_{2}$ mole fractions in scramjet combustors. ${ }^{5}$

\footnotetext{
${ }^{1}$ Graduate Student, AIAA Student Member.

${ }^{2}$ Professor, Mechanical and Aerospace Engineering Department, 1 Old Oyster Point Road, Suite 200. Associate Fellow AIAA.

${ }^{3}$ Research Scientist, Advanced Sensing and Optical Measurement Branch, MS 493, Associate Fellow AIAA.
} 
Experimental facilities have been designed to provide flow-fields relevant to CFD modelers and that can also be studied with the DP-CARS technique. ${ }^{6,7}$ An axisymmetric supersonic, combusting, coaxial jet, providing good optical access and symmetry (useful to minimize the number of measurements required to characterize the flow) was chosen for our experiment. The facility provides a center jet of "vitiated" air, reproducing the sensible enthalpy of air entering the engine of a hypersonic vehicle flying at Mach numbers between 5 and 7 . The vitiated air is the product of combustion of $\mathrm{O}_{2}$-enriched air with $\mathrm{H}_{2}$ that has the same $\mathrm{O}_{2}$ mass fraction of air, but high temperature and excess water. Nozzles with exit Mach number 1, 1.6 and 2 are available to provide flows with different compressibility. Hydrogen or ethylene co-flow is available in order to generate a supersonic combusting free jet. Two different sizes of the experimental hardware have been developed; the first has a $1 \mathrm{~cm}$ nozzle exit diameter, and flow rates compatible with a combustion diagnostic laboratory; the second was scaled up by a factor of 6.35 from the smaller device and tested in NASA Langley's Direct Connect Supersonic Combustion Test Facility (DCSCTF). Previous works describe the large scale supersonic burner, and both numerical ${ }^{8}$ and experimental ${ }^{9}$ data have been published. Numerical computations of the internal flow-field for the laboratory scale supersonic burner have been previously presented. ${ }^{10}$ Limited experimental results were presented in Ref. 11. Since those measurements were taken, many changes, reported herein, have been made to the CARS system, improving the quality of the measurements. The laboratory-scale supersonic burner offers several advantages with respect to its large-scale version. It can be operated continuously for several hours allowing acquisition of a much larger data set. In addition, it can be operated in the CARS laboratory, which remains accessible at all times during the test. The small scale allows mounting it on a translation stage, rather than translating part of the optical system. This improves the stability and reliability of the CARS system. The drawback is that the size of the CARS probe volume, typically $1-2 \mathrm{~mm}$, is too large to resolve the smallest turbulence structures in the flow.

This paper first describes the experimental set-up, providing descriptions of the laboratory-scale supersonic burner, and of the DP-CARS instrument. Then we describe the method of analyzing the data, focusing on the solution of some issues specific to the data acquired in the supersonic burner. A discussion of the DP-CARS instrument accuracy and precision determined using a Hencken burner flame as validation case is also presented. Selection of the line shape models for the theoretical spectra is discussed, and experimental data are used to calibrate the values of the third order nonresonant susceptibility and of the optical diffusion coefficient (required for measurement of $\mathrm{H}_{2}$ ). The issue of a limited dynamic range is identified and discussed, and the accuracy of the instrument in presence of camera saturation in some pixels is determined. Finally, data collected in the laboratory-scale burner for a mixing case and a combustion case are presented. The data here shown are only a portion of the entire dataset, but are representative of the capabilities of the instrument.

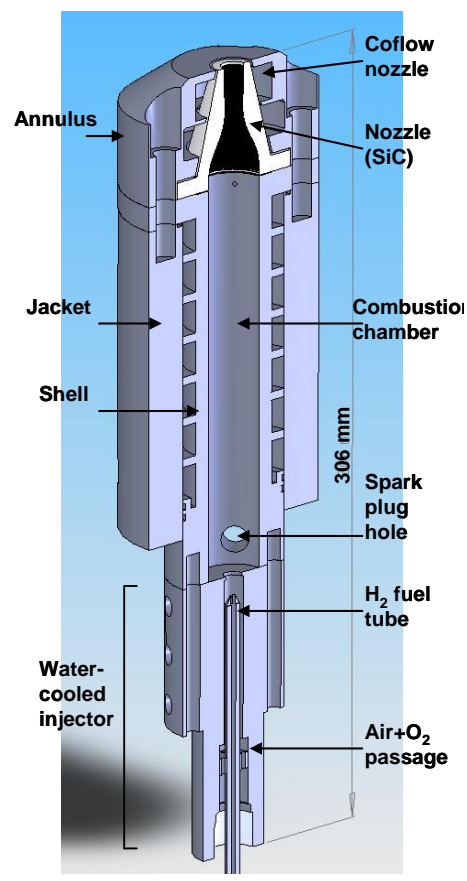

Figure 1. Schematic drawing of the laboratory scale supersonic burner.

\section{Experimental Set-up}

Figure 1 shows a schematic of the supersonic burner sectioned along the symmetry plane. It consists of a watercooled combustion chamber (burner) and a silicon-carbide (SiC) nozzle with an exit diameter of $10 \mathrm{~mm}$. An annulus surrounds the nozzle, holding it in place, and with the exterior wall of the nozzle it creates an annular, convergent "co-flow" nozzle. The internal diameter of the co-flow nozzle exit is $16 \mathrm{~mm}$ and the external is $18 \mathrm{~mm}$. The co-flow and center jets are concentric and separated by the 3-mm wide, annular base region, orthogonal to the center jet axis. The "annulus" external walls are inclined at 60 degrees to improve optical access. The burner is made of AISI 310 stainless steel, the nozzles of Hexaloy grade SA silicon carbide ( $\mathrm{SiC}$ ). The $\mathrm{SiC}$ was selected because of its elevated maximum operation temperature in air, and its large thermal conductivity (which reduced thermal stresses). Thermal insulating gaskets separate the nozzle from the stainless steel burner. The facility quickly reaches equilibrium temperature and can be operated continuously. More details on the facility can be found in Ref. 10.

Three interchangeable $\mathrm{SiC}$ nozzles with exit Mach numbers $\left(\mathrm{M}_{\mathrm{e}}\right)$ of 1, 1.6 and 2 are available. The enthalpy of the vitiated air center-jet, expressed in terms of flight Mach number $\left(\mathrm{M}_{\mathrm{f}}\right)$, can be varied between 5 and 7 by changing the flow-rates of air, $\mathrm{O}_{2}$ and $\mathrm{H}_{2}$. Data have been acquired for mixing cases in which the co-flow gas is $\mathrm{N}_{2}$, 
and combustion cases using $\mathrm{H}_{2}$ as co-flow. Combustion data were not acquired for $\mathrm{M}_{\mathrm{e}}=2$. Four Hastings flow-meters are used to regulate the mass flow-rates. The nominal accuracy of the flow-meters is $1 \%$ of the full scale, which is 25 standard liter per minute (SLPM) for the air flow, 10 SLPM for $\mathrm{H}_{2}$ and 3 SLPM for $\mathrm{O}_{2}$. Flow rates for the conditions tested are in Table 1.

In this work we show results obtained for $\mathrm{M}_{\mathrm{e}}=1.6$ and $\mathrm{M}_{\mathrm{f}}=7$ (indicated by bold typeface in Table 1), for both mixing ( $\mathrm{N}_{2}$ co-flow), and combustion $\left(\mathrm{H}_{2}\right.$ co-flow). The remainder of the data will be presented elsewhere. Pressure measurements are taken in the combustion chamber (reported in Table 1), at the exit of the nozzle and of the co-flow nozzle. A FLIR Model SC4000-MWIR infra-red (IR) camera, with sensitivity in the 3-5 $\mu \mathrm{m}$ range and an electronic exposure time set nominally to $12 \mu$ s was used to obtain IR images of the jet.

Table 1: Mass flow-rates and measured pressure for the combination of $M_{e}$ and $M_{f}$ tested.

\begin{tabular}{|c|c|c|c|c|c|c|}
\hline \multicolumn{4}{|c|}{ Vitiated Air Center-Jet Flow Rates (SLPM) } & \multicolumn{2}{|c|}{ Co-Flow Rates (SLPM) } & \multirow[b]{2}{*}{ Pressure (atm) } \\
\hline $\mathrm{M}_{\mathrm{e}} \mathrm{M}_{\mathrm{f}}$ & Air & $\mathrm{O}_{2}$ & $\mathrm{H}_{2}$ & $\mathrm{H}_{2}$ (Combustion) & $\mathrm{N}_{2}$ (Mixing) & \\
\hline 0.757 & 310.8 & 115.4 & 135.0 & 120.0 & 120 & 1.35 \\
\hline 1.007 & 426.1 & 123.9 & 185.1 & 120.5 & 120 & 1.68 \\
\hline 1.606 & 939.8 & 182.8 & 266.6 & 483.0 & 120 & 4.00 \\
\hline 1.607 & 741.9 & 221.0 & 322.3 & 419.1 & 120 & 3.88 \\
\hline 2.006 & 1273.0 & 242.0 & 361.0 & 666.0 & 120 & 7.12 \\
\hline
\end{tabular}

The CARS system is the same as described in Ref. 12, with some modifications noted below. An injectionseeded pulsed Nd:YAG laser (Spectra Physics Quanta Ray Pro-350) frequency-doubled to $532 \mathrm{~nm}$, a narrow band dye laser (Spectra Physics PDL-2) centered at $551 \mathrm{~nm}$ with a FWHM of $\sim 0.5 \mathrm{~cm}^{-1}$ and a home-built broadband dye laser centered at $603 \mathrm{~nm}$ with a FWHM of $265 \mathrm{~cm}^{-1}$, are used to generate the CARS signal. The pulse width of the $\mathrm{Nd}$ :YAG laser is $8 \mathrm{~ns}$ and the repetition rate is $20 \mathrm{~Hz}$. The output of the Nd:YAG laser, approximately $900 \mathrm{~mJ}$, is divided three ways: $170 \mathrm{~mJ}$ to pump the broadband dye laser, $415 \mathrm{~mJ}$ for the narrowband dye laser, and $280 \mathrm{~mJ}$ for the CARS green beam. The three lasers are located on a mobile cart, and the beams are relayed to the measurement volume through several relay mirrors. Laser energies at the measurement volume are approximately 90, 30 and 20 $\mathrm{mJ}$ for the Nd:YAG, the narrowband and the broadband dye laser, respectively. We chose a planar BOXCARS phase-matching geometry, with the narrowband and the broadband dye lasers collinearly overlapped, and a crossing angle between dye laser beams and the Nd:YAG beam of 4.5 degrees. Dichroic mirrors are used to combine the two dye lasers and to separate the Nd:YAG laser from the CARS signal centered at $490 \mathrm{~nm}$. Two distinct lenses focus the beams at the measurement volume. A $600 \mathrm{~mm}$ focal length spherical lens focuses the Nd:YAG beam; the lens is tilted so that by introducing astigmatism, it shapes the focal spot at the beam crossing to a 4:1 axis ratio ellipse with the major axis orthogonal to the plane formed by the three laser beams. This, combined with the planar BOXCARS phase-matching geometry, helps in reducing beam steering effects in flows that are turbulent and have large density gradients. ${ }^{13}$ The superimposed dye laser beams are focused by an $850 \mathrm{~mm}$ focal length spherical lens. The long focal length of the two lenses insures that the laser irradiances are below the Stark broadening and stimulated Raman pumping thresholds determined in Ref. 12. Two beam viewing systems ${ }^{14}$ are used to image a cross section of the three beams in the vicinity of the measurement volume. The first beam viewing system is placed before the measurement volume and consists of a wedged glass plate placed in the path of the beams at 45 degrees which creates an image of the beam crossing. A microscope objective lens, filters, and a CCD camera are used to detect the image of the crossing. This beam viewing system is used to accurately focus and cross the beams so that the CARS signal is maximized. The wedged glass plate is mounted on a flip mount and removed during data acquisition to maintain the crossing location. The second beam viewing system is placed after the measurement volume, and differs from the first by an achromatic spherical lens, that refocuses the three beams on a microscope objective. The system is used to monitor the position and the focusing of the beams during a test, and evaluate the effect of beam steering and temperature changes in the optics. At the measurement volume the three beams interact and generate a coherent laser-like beam containing a broad CARS spectrum centered at $490 \mathrm{~nm}$. A series of dichroic mirrors and filters is used to separate the signal from the Nd:YAG beam. The signal is then focused by a spherical lens on the input slit of a one-meter McPherson spectrometer equipped with a 2400 lines/mm grating. The CARS signal is dispersed by the spectrometer and recorded on a CCD camera mounted at the exit. The camera (Princeton Instruments PIXIS 100B) has a detector of 1340x100 pixels. For this series of tests the CCD is binned into three rows to reduce the read-out time and to allow data acquisition at $20 \mathrm{~Hz}$. Binning also increases the signal-to-noise ratio. The CARS signal is only in the central bin; the upper and lower bins are used for background subtraction as illustrated in more details in the next section. The dynamic range of the camera is 16 bits, limiting the maximum 
number of counts to 65535 . When the CARS signal exceeds this threshold, it can be lowered by reducing the energy of the Nd:YAG laser; this is accomplished by rotating the angle of a half-wave plate followed by a polarizer, placed on the laser cart. The half-wave plate is mounted on a remotely controlled rotation stage. More details on our approach for dealing with the dynamic range limitation are in Section IV-D.

The laboratory-scale supersonic burner is mounted on a three-axis translation stage, which allows changing the position of the measurement volume with respect to the flame. The coordinates of the data points presented in this works are in a reference system having the origin at the center of the nozzle, at its exit plane. The $z$ axis is orthogonal to the nozzle exit plane, the $y$ direction parallel to the Nd:YAG laser beam. Radial profiles were performed by scanning in the $x$-direction (perpendicular to the laser beams). The origin of the reference system is determined experimentally immediately before the data acquisition, with the supersonic burner running at the test conditions. CARS signal intensity in the $\mathrm{O}_{2}$ spectrum is recorded at several locations along the $x$ and $y$ axis, $1 \mathrm{~mm}$ from the nozzle exit. Since $\mathrm{O}_{2}$ is not present in the co-flow, this allows determining the location of the nozzle axis, with respect to the CARS measurement volume. The process is automated, and is performed daily before starting the data acquisition.

The data acquisition software is programmed so that once the acquisition is started no further input from the operator is required. Unfortunately full automation was not possible in real test conditions. The supersonic combusting free jet slowly increases the temperature of all the optical elements. This causes a drift of the laser beams' alignment with time, degrading the beam overlap, and consequent signal reduction. Such drift was monitored using the beam viewing system placed after the measurement volume. Periodically the test had to be interrupted, so that the relative position between the lasers could be restored. We would use the Nd:YAG beam as reference, and restore the dye lasers position with respect to this beam by remotely controlling two mirrors (mounted on New-Focus picomotors mounts); one is placed on the laser cart and adjusts the broadband dye laser, the other which adjusts the superimposed dye lasers is placed immediately before the focusing lens. Note that a sideways drift of the location of the measurement volume with time was not corrected by this system, though the error induced by this drift is estimated to be a fraction of the lenght of the measurement volume.

\section{Data Analysis}

The raw signal collected by the CCD camera contains not only the CARS signal but also contributions from other sources that have to be removed. A major contribution is the camera dark noise. This contribution is almost identical in all three bins, (within 1 count); shot-to-shot variations in each binned pixel have a standard deviation of 4.5 counts. The mean value typically changes $1-2$ counts with time, (depending on the CCD temperature) as long as the camera has been warmed up for few hours before the test. Ambient light, prevalently from the room illumination can reach the camera. Barriers, built around the camera and the relay mirrors limit this contribution to 1-2 counts above the dark noise level. With the current set-up some light from the Nd:YAG flashlamps reaches the camera. The light is collinear with the Nd:YAG beam, and therefore partially overlapped to the CARS signal itself. The portion of the light at the same wavelength of the CARS signal reaches the camera's central bin. The contribution is not uniform over the CCD, but has a well-defined spectral distribution. Use of Pelin-Broca prisms on the cart reduces its peak amplitude to $\sim 20$ counts. This emission passes through and is altered by the half-wave plate and the polarizer used to reduce the signal intensity in the presence of detector saturation. To take into account these contributions 250 background spectra are taken for each value of the half-wave plate angle used during the test, by blocking the narrowband dye laser on the cart. For each test run, we subtract, bin by bin, from the raw signal the average of the background obtained with the same angle of the half-wave plate used for that specific run.

The procedure described above does not remove the contribution from the flame luminescence. This contribution depends on the location of the flame, and therefore could not be taken into account by subtracting a reference spectrum. The contribution from the flame is present in all three bins, but is higher in the central bin. The ratio of the flame luminosity between bins stays constant when moving the flame location. To take into account the flame luminosity contribution we subtract from the central bin the average of the bin containing the least signal, multiplied by a scaling factor that takes into account the different light distribution in the three bins. Flame luminosity is an issue only for the combustion cases of the supersonic jet. Mean values up to 20 counts have been observed.

Data acquisition in the supersonic jet typically took 4 hours. Small changes with time in the background spectra are taken into account by interpolating between spectra taken before and after the test. Tests in the Hencken burner were generally completed within an hour, and time variation in the background was neglected. CARS spectra have resonant and non-resonant parts. In DP-CARS absolute mole fraction measurements are determined by the ratio between the resonant and the non-resonant portion of the spectrum. The non-resonant baseline intensity ranges from 
15 counts to a few hundred; therefore it is comparable to the contributions from the flashlamps and the flame luminescence. Correct removal of spurious light from the non-resonant baseline is therefore critical for accurate measurements of absolute mole fractions.

Beam steering and changes in temperature of the optics can move the location of the CARS signal on the camera. A pre-processing algorithm shifts the spectra so that the location where the $\mathrm{N}_{2}$ band is $20 \%$ of its peak values is always at the same pixel location. This feature was introduced by Tedder ${ }^{9}$ for measurements in the large scale supersonic jet. For this experiment, typical movements of the spectra on the CCD were limited to a couple of pixels.

The CARS signal, obtained by removing the background from the raw signal, needs to be normalized by the excitation profile of the broadband dye laser. This profile is obtained by averaging 500 single shots of non-resonant CARS signal obtained in a flow of argon, which has no Raman resonances in the probed spectral region. Nonresonant spectra are collected at the beginning and at the end of the test day. During the several hours required for data acquisition, the measured non-resonant spectrum changes its peak wavelength and its shape. The change is due to several factors, including changes in the temperature of the dye, solvent evaporation, laser misalignment and changes in the intensity of the Nd:YAG beams that pump the dye cells. The preprocessing algorithm interpolates the non-resonant spectrum assuming that the changes are linear with time - an assumption that was shown to be sufficient for measurements in Hencken burner. Over the four hours of a typical test in the supersonic jet, the change in the non-resonant spectrum peak wavelength is not monotonic; therefore the linear approximation can cause large errors. Unfortunately this was not considered at the time of data acquisition, and only two non-resonant spectra are available, one before and one after the whole data set is acquired. Figure 2a shows the fit to the theory of an average spectrum obtained with the non-resonant referencing described above. The left side of the spectra is lower than the theory, the right side higher. This error is a typical consequence of an erroneous referencing (due to an incorrect shift in the Gaussian shape of the non-resonant spectrum) and can be corrected by shifting the non-resonant spectrum by an additional 35 pixels as shown in Fig. 2b. Visual inspection of all data is impractical given the large amount of data collected.

In Ref. 5 O`Byrne faced a similar problem and determined the position of the non-resonant spectrum by fitting the middle one third of each run for three or more values of the center wavelength of the nonresonant spectrum, and determining the optimal values as the one minimizing the fitting residual. This approach is time consuming since it requires multiple fits of each dataset; given the large amount of data we chose a less accurate but faster approach. Figure 2a shows that an incorrect non-resonant shift causes an erroneous slope in the non-resonant baseline. We use an algorithm that evaluates the ratio of the average experimental spectra in the $2085-2090 \mathrm{~cm}^{-1}$ and in the $2240-2245 \mathrm{~cm}^{-1}$ region. This ratio is a function of temperature and composition, but does not change much in the range of temperature and concentration expected. For the combustion case, values are $\sim 1.04$ for the nozzle exit composition and temperature, 0.84 for the pure $\mathrm{H}_{2}$ or $\mathrm{N}_{2}$ co-flow, and 0.95 for air, based on theoretical spectra obtained with CARSFT. The ratio is mostly sensitive to changes in the $\mathrm{O}_{2}$ and $\mathrm{H}_{2} \mathrm{O}$ mole fractions. The algorithm first determines if the presence of $\mathrm{O}_{2}$ is significant (peak of the $\mathrm{O}_{2}$ spectrum greater than $10 \%$ of the total spectrum peak, before normalization by the non-resonant), to choose which value of the average ratio to use $\left(0.84\right.$ if $\mathrm{O}_{2}$ is negligible, 1.04 otherwise). Then it computes the ratio in the experimental average data when normalized by the interpolated non-resonant spectrum, and by the same spectrum shifted by \pm 60 pixels. Finally, it fits the computed average ratios with a quadratic function to
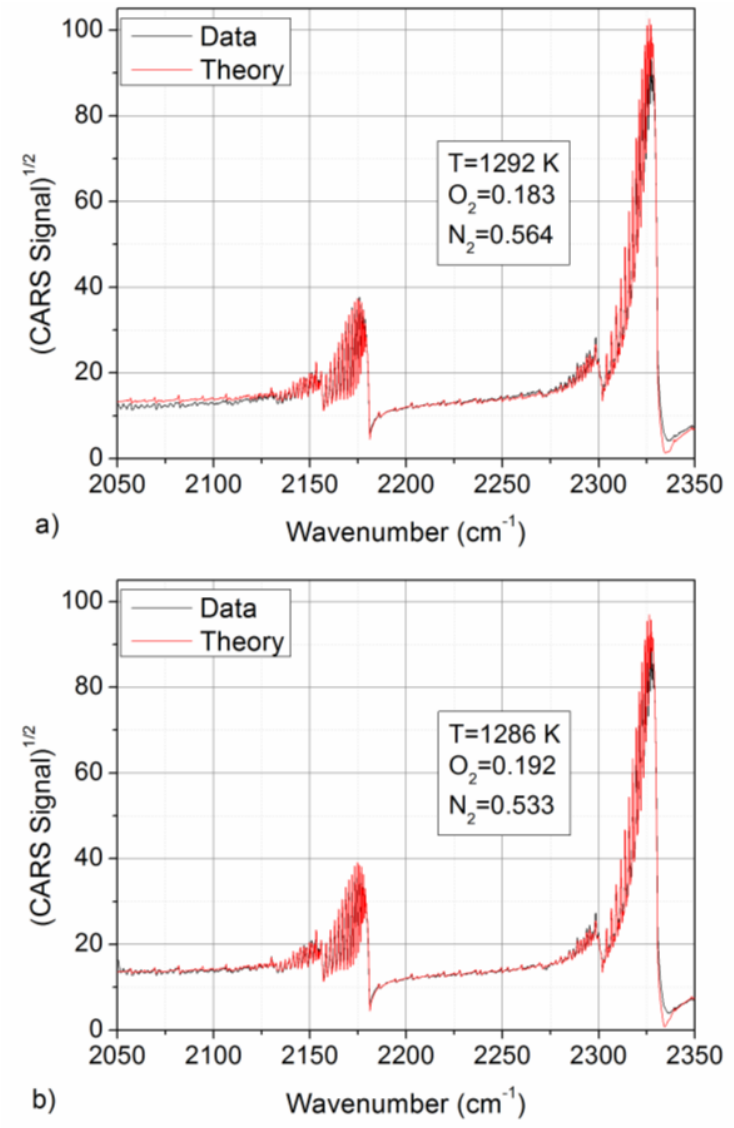

Figure 2. Experimental spectra and theoretical fit before (a) and after (b) shifting the excitation profile. 
determine the shift necessary so that the ratio in the experimental data matches the desired value.

The main limitation of this approach is that the theoretical ratio is not known a priori and is approximated to one of two set values. For the range of temperature and concentrations expected in the test, the ratio can take values in the 0.84-1.1 range; for a given spectrum the difference in the non-resonant shift computed by the algorithm using the two limit values is 32 pixels, therefore an uncertainty of \pm 16 pixels is caused by not knowing the exact composition and temperature in the preprocessing. Errors in background subtraction can lead to bigger errors when this algorithm is used because they affect the baseline, and therefore the ratio used to determine the non-resonant shift. This is especially problematic for spectra taken in colder gases, where the laser irradiance has been strongly reduced to avoid detector saturation in the spectral peak. For this reason the algorithm uses the shift determined for the previous set of spectra for spectra having less than 25 counts on the non-resonant baseline. The algorithm returns the shift in the non-resonant spectra up to 100 pixels, but the change in the shift computed for two consecutive runs is within 30 pixels. In terms of measured mole fraction, a 30 pixel shift corresponds to $~ 5 \%$ change in the measured absolute mole fraction, $10 \%$ in the relative mole fraction, and $2 \%$ in the temperature. Shot-to-shot referencing schemes as used in Ref. 15 might remove this source of uncertainty and greatly improve the instrument accuracy.

The preprocessed spectra are then square rooted and analyzed using the fitting algorithm developed by Cutler. ${ }^{16}$ The algorithm requires a library of theoretical spectra generated with a version of CARSFT ${ }^{17}$ modified by Hancock ${ }^{18}$ and O'Byrne ${ }^{19}$ for using with DP-CARS. Theoretical spectra were modeled using the Tipping-Buoanich ${ }^{20}$ in place of the James-Klemperer ${ }^{21}$ model originally used by CARSFT for the Herman Wallis factor, to improve the theoretical modeling of $\mathrm{N}_{2}$ and $\mathrm{O}_{2}$ at higher temperatures. ${ }^{22}$ In addition to the molecular parameters, CARSFT requires the width of the pump laser, the width of the instrument function (mainly owing to the spectrometer), the width of the probe laser, and the offset in wavenumbers between the pump and probe beams. The Nd:YAG laser is injection seeded, and its width is considered infinitesimal in the spectral convolution. The instrument function is assumed to be Gaussian and its width is determined with CARSFT as a result of a fitting over the $\mathrm{O}_{2}$ portion of the spectrum. This region is chosen because it is independent of the narrowband dye laser width. The width of the narrowband dye laser is determined by fitting this parameter in the $\mathrm{N}_{2}$ region, using the value of the instrument function determined in the previous step. The offset between the Nd:YAG laser is also determined using CARSFT, so that the offset between the $\mathrm{N}_{2}$ and $\mathrm{O}_{2}$ portions of the spectra in the theory match the experiment. During the long tests (up to 4 hours) for acquiring data in the supersonic burner, the frequency of the narrowband dye laser was changing in time, as a consequence of the temperature increase in the room. The shift was monotonic, and changes up to 0.8 wavenumbers were observed for the combustion cases. For this reason for each test day four libraries, with different values of the offset between the two pumps, were generated, one for each hour of testing. Details on the criteria to generate the libraries and on the fitting algorithm can be found in Ref. 16.

\section{Dual-Pump CARS Instrument Characterization}

Measurements in a Hencken burner flame were performed to validate and calibrate the DP-CARS instrument. The Hencken burner produces a steady, nearly adiabatic flat flame, often used for calibration and validation of nonintrusive optical diagnostics techniques. ${ }^{23}$ Similar measurements, obtained at NASA Langley with earlier versions of the DP-CARS instrument are reported in Refs. 5, 9, 11. Four Tylan (model FC-280) mass flow controller were available to control the flow rate of $\mathrm{H}_{2}$, air and $\mathrm{O}_{2}$ to the burner. Each flow controller can provide flow rates up to 30 SLPM with an accuracy of 0.3 SLPM (1\% of full scale). One flow-controller is used to regulate the $\mathrm{H}_{2}$, one is dedicated to the $\mathrm{O}_{2}$ flow rate, and the remaining two provide control over the air flow. The flow-meters are set to produce mixtures of air and $\mathrm{H}_{2}$ with equivalence ratios in the 0 to 3.5 range. Air flow-rates are varied between 17 and 60 SLPM, and only one flow controller is needed when the flow rate is below 30 SLPM, that is for $\Phi>2 . \mathrm{H}_{2}$ flow rates are in the 2.5 to 25 SLPM range. Total flow rates are in the 40 to 75 SLPM range. The flow rates are doubled with respect to what was used in Refs. 5 and 9; the higher flow-rates ensure that the flame is slightly lifted from the burner surface so that heat losses are reduced and the flame is near-adiabatic; in addition it allows for operation of the $\mathrm{H}_{2}$ flow controller closer to full scale reducing the effects of inaccuracies of the flow-meter. In addition to air- $\mathrm{H}_{2}$ mixtures, mixtures enriched with $\mathrm{O}_{2}$ have also been considered, with flow rates chosen so that the products of combustion have $21 \% \mathrm{O}_{2}$ by mole fraction. Such test cases provide additional validation points in conditions more similar to the vitiated air mixture that constitutes the center jet produced by the laboratory-scale burner. The enriched mixtures are limited to equivalence ratio of 0.26 , corresponding to a temperature of $1400 \mathrm{~K}$. The additional oxygen moves the flame closer to the burner surface, and for higher equivalence ratios the larger heat transfer violates the assumption of near adiabatic flow, and can damage the honeycomb structure of the burner. 


\section{A. Non-uniformities in the Hencken burner flame}

Previous measurements showed some spatial non-uniformity in the flame produced by the Hencken burner available at NASA Langley. For the current work, a more detailed survey of the flame produced from the burner has been performed. Two equivalence ratios $(\Phi)$ have been considered, one fuel lean $(\Phi=0.3)$, and the other fuel rich $(\Phi=2)$. For each equivalence ratio, data have been collected in a vertical plane, orthogonal to both the burner surface and the direction of the Nd:YAG laser, and in a horizontal plane, parallel to and $40 \mathrm{~mm}$ above the burner surface. The test matrix for the vertical plane has 7 locations along the vertical axis and 11 along the horizontal direction. The first axial location is $5 \mathrm{~mm}$ from the burner, the last $80 \mathrm{~mm}$. The test matrix for the horizontal plane covered a $20 \times 20 \mathrm{~mm}$ square region, with 11 data points in each direction, for a total of 121 distinct points. The dots in Figs. 3 and 4 represent the measurements locations. Each point is visited twice, and 250 single-shot spectra have been acquired each time. The two replications allow verification of steady operation. The 250 repetitions are used to determine average and standard deviation at each location. The order of the test matrix for each plane and equivalence ratio was randomized. Figure 3a shows contour plots of average temperature in the vertical plane for the fuel lean case. The average is computed over the 250 repetitions and the two replications. Data collected $5 \mathrm{~mm}$ from the burner surface shows a very hot region $(\sim 1400 \mathrm{~K}) 1 \mathrm{~mm}$ from the center of the burner, coupled with a much colder region $(\sim 1000 \mathrm{~K})$ on its right. This is evidence of an inhomogeneous distribution of the local equivalence ratio caused by the obstruction of some fuel tubes. Moving downstream, mixing makes the temperature more uniform, but $80 \mathrm{~mm}$ from the surface there is still evidence of the initial non-uniformity. Figure 4a shows the average temperature in the horizontal plane $40 \mathrm{~mm}$ from the burner. The temperature distribution is even more inhomogeneous than shown in the vertical plane. The hot region is coupled with a much colder one (less than $900 \mathrm{~K}$ ) that is not visible in the vertical plane image.
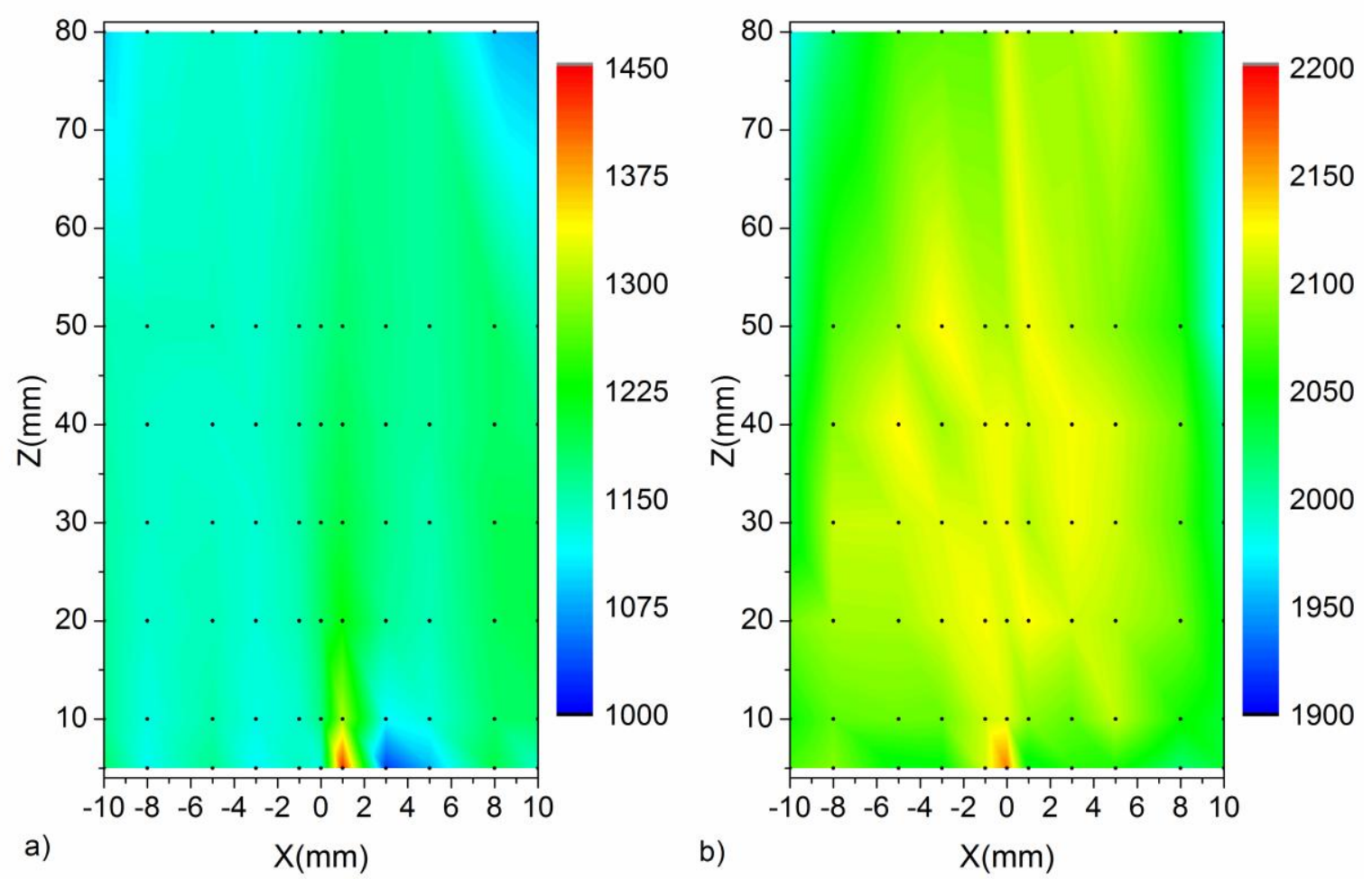

Figure 3. Temperature contour plot in the vertical plane ( $y=0)$ for a) $\Phi=0.3$ and $b$ ) $\Phi=2$.

Figures $3 \mathrm{~b}$ and $4 \mathrm{~b}$ are obtained for an equivalence ratio of 2 . Again there is a strong non-uniformity $5 \mathrm{~mm}$ from the burner surface, with variations in the equivalence ratio that are consistent with what observed in the fuel lean case. Notice that for equivalence ratios closer to 2, reducing the equivalence ratio increases the temperature. For given change in the equivalence ratio, the resulting temperature variation is larger for fuel lean mixtures than fuel rich. This explains the smaller temperature range observed for $\Phi=2$ than for $\Phi=0.3$. Mole fraction measurements of 
$\mathrm{H}_{2}$ are consistent with the temperature measurements, and the range observed is comparable to the range of $\mathrm{O}_{2}$ observed for the fuel lean case. Although there are very large variations $5 \mathrm{~mm}$ from the surface, the flame becomes much more uniform further downstream. The survey of the horizontal plane shows significantly less variations than the fuel lean case.
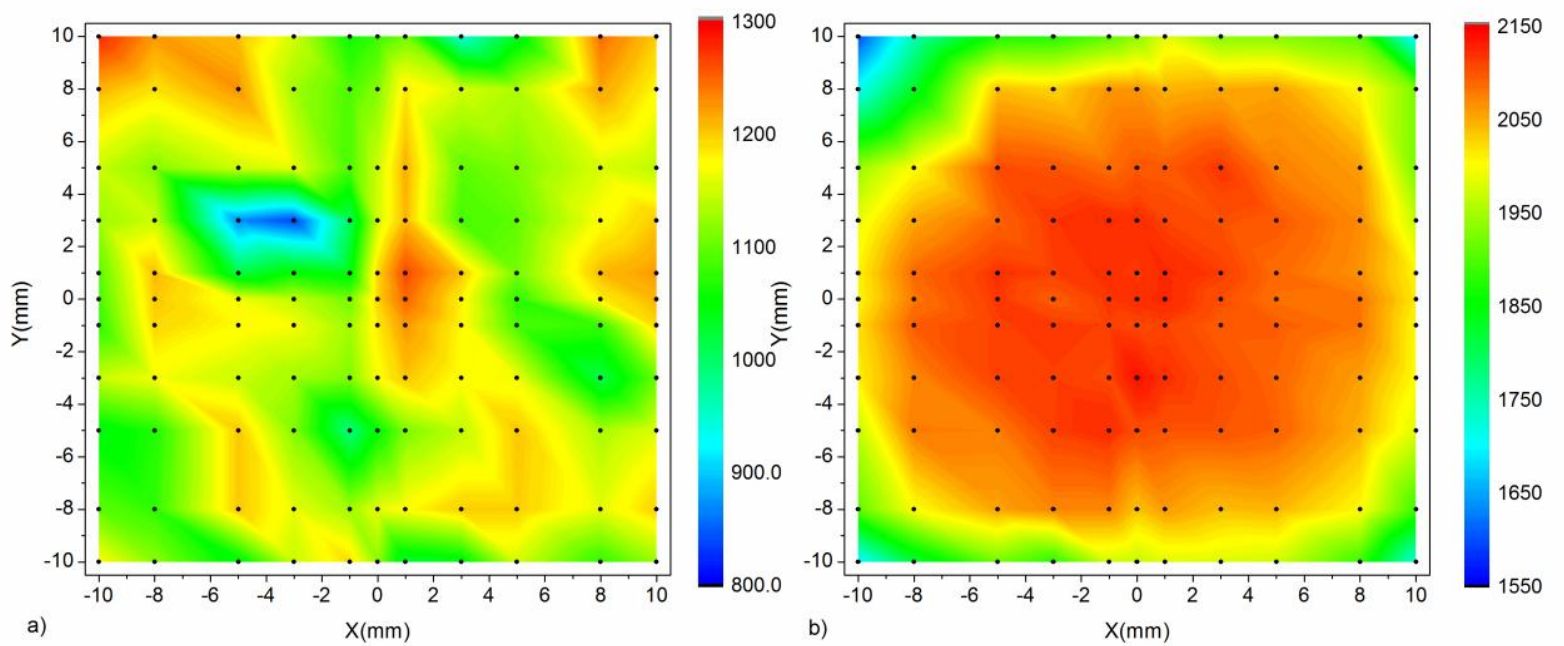

Figure 4. Temperature contour plot in the horizontal plane $(\mathrm{z}=40 \mathrm{~mm})$ for a) $\Phi=0.3$ and $\mathrm{b}) \Phi=2$.

Based on these plots it appears that it would be best to take validation measurements as far downstream as possible. However, moving the probe volume downstream weakens the hypothesis of adiabatic flame, because of the cooling effects of radiation. In addition, $\mathrm{N}_{2}$ from the co-flow increasingly penetrates the flame further downstream, reducing the temperature, affecting the mole fractions, and causing a large increase in the standard deviation. The study does not show a region that is both uniform and for which the assumption of adiabatic, laminar flame is satisfied. Lacking a better test-medium, the validation study was performed in the Hencken burner flame, but these non-uniformities are taken into account when interpreting the results. The data presented in the next section were collected $50 \mathrm{~mm}$ above the burner surface at the center of the burner.

\section{B. Instrument accuracy}

The instrument accuracy is determined by comparing CARS measurements in the Hencken burner to theoretical calculations for several values of the equivalence ratio. The calculations, which assume chemical equilibrium, have been performed using the Chemical Equilibrium Analysis code (CEA) from NASA Glenn Research Center. ${ }^{17}$ Theoretical calculations have been conducted for nominal flowrates and for two limiting cases that bound the inaccuracies due to mass flow meters. One limit case is obtained by subtracting 0.3 SLPM from the flow-rate of $\mathrm{H}_{2}$ and adding 0.3 SLPM to the flow-rate provided by each of the air flow-meters. It is denoted as "leaner" limit case since it produces a leaner mixture than the nominal case. Vice-versa, the "richer" limit case is obtained by subtracting 0.3 SLPM from each oxidizer flow rate, and adding 0.3 to the $\mathrm{H}_{2}$ flow rate.

Figure 5 shows the CARS measured temperature (red circles) as function of the equivalence ratios. The continuous line are the computed values for the nominal equivalence ratio, the dashed lines are for the leaner and richer limit cases. Fitted temperatures are

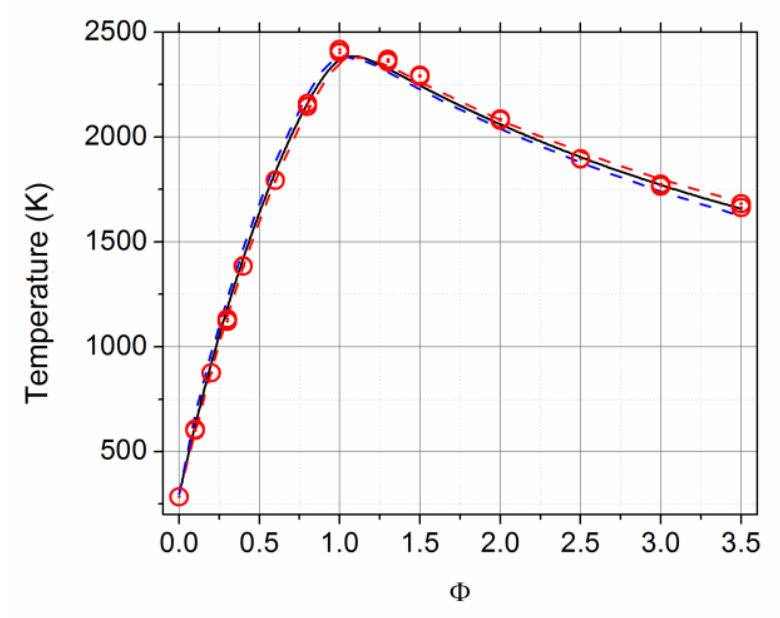

Figure 5. Measured (red circles) and computed (continuous black line) temperature as function of the equivalence ratio $\Phi$. 
within $60 \mathrm{~K}$ of the expected nominal temperature over the entire range, and within $20 \mathrm{~K}$ for $\Phi>2$. Measured temperatures are systematically low for fuel lean mixtures, and high for rich mixtures with $\Phi<2$. This is evidence of a systematic difference between the local equivalence ratio and the global (average) which we attribute to non-uniformity described previously. Hancock ${ }^{23}$ pointed out that the overall shape of the CARS fitted temperature provides a more accurate estimate of the equivalence ratio than one computed based on the mass flow-rates. For the remainder of this section, we use the composition corresponding to the measured temperature for an $\mathrm{H}_{2}$-air flame in chemical equilibrium. By doing so, we remove the uncertainty of the equivalence ratio, but we make a potential error by assuming the temperature measurement to be exact.

Mole fraction measurements are sensitive to the choice of the line shape model and to the values of the third-order non-resonant susceptibilities. In previous

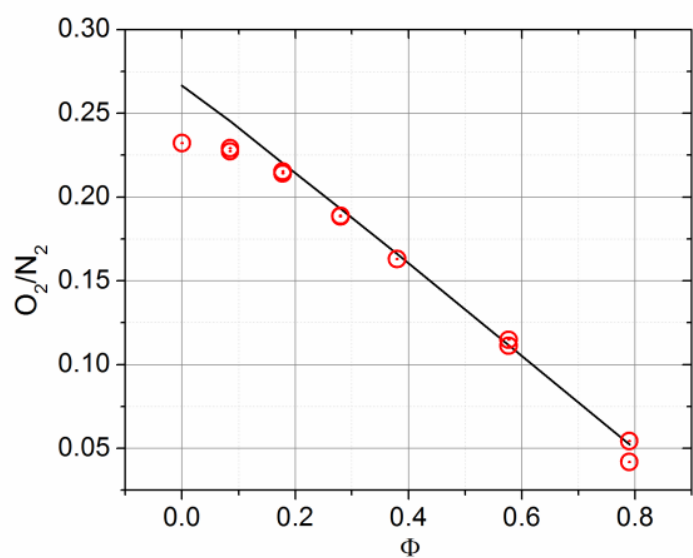

Figure 6. $\mathrm{O}_{2} / \mathrm{N}_{2}$ ratio as function of the equivalence ratio $\Phi$.

works, ${ }^{5,9,11}$ the Voigt model was used for the line shape of all species, and values from Ref. ${ }^{24}$ were used for the third order non-resonant susceptibilities. In order to improve the accuracy of the mole fraction measurements we considered the use of different line shape models, and calibrated the values of the third-order non-resonant susceptibility for all the major species.

The relative mole fractions are independent of the third-order non-resonant susceptibilities, but systematic error in their measurements might be caused by inadequate line shape models. Figure 6 shows the ratio of $\mathrm{O}_{2} / \mathrm{N} 2$ measured for fuel lean mixtures using the Voigt model for both $\mathrm{N}_{2}$ and $\mathrm{O}_{2}$. The error in the mole fraction ratio is below $4 \%$ for $\Phi \geq 0.2$, corresponding to temperatures greater than $900 \mathrm{~K}$. At lower temperatures the error is significantly larger, $8 \%$ at $600 \mathrm{~K}$ and $13 \%$ at $300 \mathrm{~K}$. The results suggest that the line shape model might be inadequate at lower temperatures.

In Q-branch spectra for low values of the rotational quantum number $J$ the ro-vibrational lines are overlapped and computation of the line shape as an isolated line undergoing pressure broadening is no longer adequate. Collisional narrowing, "which causes a coalescence or collapse of adjacent transitions toward a frequency center of gravity determined by the most populous rotational state" 25 becomes significant. This phenomenon needs to be taken into account at low temperatures, where only the lower rotational states are populated. For higher values of $J$, the line splitting increases and collisional narrowing is no longer significant. The Voigt model includes the pressure and the Doppler broadening, but not the collisional narrowing. Figure 7 shows the fit of the average of 500 spectra collected in room air, to a theoretical spectrum computed using the Voigt model for the line shape of $\mathrm{O}_{2}$ and $\mathrm{N}_{2}$. The fit is good, except in proximity of the $\mathrm{N}_{2}$ and $\mathrm{O}_{2}$ band-head, hence confirming that the issue is limited to the lowest $\mathrm{J}$ numbers. There is the

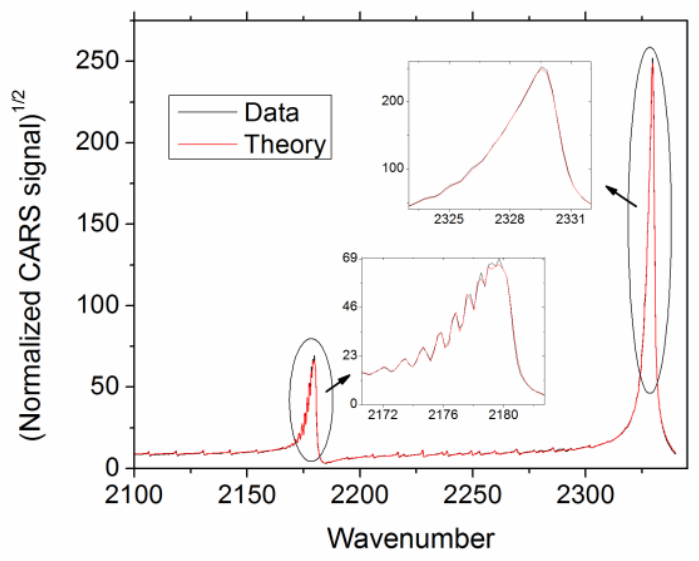

Figure 7. Fit of an average of 500 single shot spectra collected in ambient air to a theoretical spectrum computed using the Voigt model. possibility of improving the accuracy at low temperatures by implementing line shape models that include the collisional narrowing effect such as the modified exponential gap model. At the current time the model is not fully implemented in CARSFT, therefore we used the Voigt model for the line shape of $\mathrm{O}_{2}$ and $\mathrm{N}_{2}$.

When using the Voigt line shape model for all species, as in Refs. 5 and 9 the measured ratio of $\mathrm{H}_{2} / \mathrm{N}_{2}$ is systematically high. For $\mathrm{H}_{2}$ at atmospheric pressure, motional (Dicke) narrowing is significant and the Galatry model, rather than the Voigt, is recommended. ${ }^{26}$ The Galatry model requires the knowledge of an additional quantity, the motional narrowing parameter $\beta$, which is related to the optical diffusion coefficient $D_{o}$ via the relation: 


$$
\beta=\frac{k T}{m D_{o}}
$$

where $k$ is the Boltzmann constant, $m$ the molecular mass, and $T$ the temperature. Units of $\beta$ are $\mathrm{cm}^{-1} / \mathrm{atm}$. Several studies show $D_{o}$, although of the same order of magnitude, differs from the conventional mass diffusion coefficient $D_{m} \cdot{ }^{27-29}$ The optical diffusion coefficient determines the ratio of the measured $\mathrm{H}_{2}$ and $\mathrm{N}_{2}$. The ratio does not depend on the non-resonant third order susceptibility. Given the uncertainty on this parameter, we calibrated it to minimize systematic errors in the measurements. For each fuel-rich condition tested in the Hencken burner we determined the optical diffusion coefficient that returns the correct ratio of $\mathrm{H}_{2}$ and $\mathrm{N}_{2}$. The values so determined are fitted to an exponential function of the temperature and input to CARSFT while generating the theoretical library. The calibration ensures that the instrument returns an accurate $\mathrm{H}_{2}$ to $\mathrm{N}_{2}$ ratio for the temperature and mole fraction tested with the Hencken burner, but problems may arise when conditions vary significantly from those produced by the Hencken burner. The calibration is a function only of temperature and if the composition in the supersonic burner, for given temperature, differs significantly from what is observed in the Hencken burner, the calibration may be in error, affecting the accuracy of the composition measurement. Also the calibration assumes that the $\mathrm{N}_{2}$ line shape model is correct. This was based on the observation of good accuracy in terms of the $\mathrm{N}_{2} / \mathrm{O}_{2}$ ratio for fuel lean mixtures at high temperature. Note that errors in the ratio of the $\mathrm{H}_{2} / \mathrm{N}_{2}$ for the data presented in Refs. 5 and 9 cannot be explained by the choice of line-shape model (or optical diffusion coefficient). Stark broadening ${ }^{12}$ of the $\mathrm{H}_{2}$ lines, was likely responsible of the erroneous measurements of the $\mathrm{H}_{2} / \mathrm{N}_{2}$ ratio reported in those previous works.

DP-CARS is capable of obtaining absolute mole fractions of all major species in an air- $\mathrm{H}_{2}$ flame, provided a knowledge of the third order non-resonant susceptibility of all the major species, both resonant and non-resonant. Accuracy of the mole fraction measurement is obviously limited by the degree to which the non-resonant background is known. Unfortunately these parameters are not well known, and although several measurements have been reported, ${ }^{24,30-35}$ they are not consistent, and have large uncertainties $(10 \%)$. For this work we calibrated the third order non-resonant susceptibilities of all major species to optimize the accuracy of the absolute mole fraction measurements. The calibration was performed in two steps; first we calibrated the third order nonresonant susceptibilities for $\mathrm{N}_{2}, \mathrm{O}_{2}$ and $\mathrm{H}_{2} \mathrm{O}$ based on the fuel lean data (excluding test conditions for which the error on the $\mathrm{O}_{2} / \mathrm{N}_{2}$ ratio is above $10 \%$, that is $\Phi=0$ and $\Phi=0.1$ ); then we determine the $\mathrm{H}_{2}$ third order nonresonant susceptibility from the fuel rich data.



Figure 8. Measured (symbols) and computed (continuous line) mole fraction as function of the equivalence ratio $\Phi$. Calibrated values of the third order non-resonant susceptibilities are 7.85, 7.79, 19.74 and 10.02 (in units of $10^{-18}$ $\mathrm{cm}^{3} /$ (erg amagat)) for $\mathrm{N}_{2}, \mathrm{O}_{2}, \mathrm{H}_{2} \mathrm{O}$ and $\mathrm{H}_{2}$, respectively. Except for $\mathrm{H}_{2}$, the calibrated values are within the uncertainty range (10\%) provided with the measurements from Hahn ${ }^{34}$ The value determined for $\mathrm{H}_{2}$ is $70 \%$ larger than what measured by Hahn, ${ }^{34}$ and $16 \%$ larger than the highest measurement we found in literature ${ }^{35}$ This suggests that there might be additional issues in line-shape modeling of the fuel-rich mixtures that are being corrected by selection of third order non-resonant susceptibility of $\mathrm{H}_{2}$. Without this correction, errors up to $3 \%$ were observed for $\mathrm{N}_{2}$ and $\mathrm{H}_{2}$. The calibration does not provide measurements of the optical diffusion coefficient or the third-order nonresonant susceptibilities, but values that are optimized for this instrument to correct for other deficiencies in the modeling, and are valid only in the neighborhood of the conditions for which they have been determined.

Figure 8 shows the measured mole fractions (symbols) of $\mathrm{O}_{2}, \mathrm{~N}_{2}$ and $\mathrm{H}_{2}$ as a function of the equivalence ratio. Continuous lines are the computed values based on an equivalence ratio corresponding to the measured temperature. The calibration provides an overall reduction of the systematic error. The largest errors are at low temperature (below $800 \mathrm{~K}$ ) and are probably caused by not including the collisional narrowing in the line shape model. The measured $\mathrm{O}_{2}$ mole fraction is $1.2 \%$ lower than expected at $300 \mathrm{~K}$ and $0.7 \%$ lower at $600 \mathrm{~K} ; \mathrm{N}_{2}$ mole fractions are $3.5 \%$ and $2 \%$ higher at room temperature and at $600 \mathrm{~K}$, respectively. Errors are reported as a percentage of the total composition, not of the measured value. At higher temperatures, the accuracy significantly improves, with errors in the $\mathrm{O}_{2}$ concentration that are below $0.5 \%$ (systematically low) and errors in $\mathrm{N}_{2}$ below $1.5 \%$ (systematically high). $\mathrm{H}_{2}$ 
mole fractions are within $1 \%$ of the total composition. The accuracy is significantly improved with respect to what is reported in Ref. 5 (errors in $\mathrm{N}_{2}, \mathrm{O}_{2}$ and $\mathrm{H}_{2}$ below 2.6, 2.4 and 5\% of total composition) and in Ref. 11 (errors in $\mathrm{N}_{2}$, $\mathrm{O}_{2}$ and $\mathrm{H}_{2}$ below $8,3.1$ and $7 \%$ of total composition). Removal of some of the experimental errors that may have affected previous measurements such as high irradiance effects, and improvements in the modeling (more accurate Herman-Wallis factors, better line shape model selection, and calibration of the non resonant susceptibilities) are responsible for the improved accuracy.

\section{Instrument precision}

In the study of turbulent flows, a high precision is desirable since it determines the lowest amplitude of fluctuations that can be detected. Instrument precision also affects the amount of data that needs to be obtained to meet measurement uncertainty criteria. The instrument precision needs to be carefully characterized, so that fluctuations in the flow can be distinguished by those caused by the instrument. Standard deviation, computed from several single-shot measurements in an oven or a calibration flame, is often used as a metric for the instrument precision., ${ }^{2}, 15$, 23, 36-42 Commonly reported single-shot temperature standard deviations for $\mathrm{N}_{2}$ ro-vibrational CARS are 25$30 \mathrm{~K}$ at $300 \mathrm{~K} \cdot{ }^{36-38,40}$ The relative standard deviation, defined as the ratio of the standard deviation to the mean measured temperature, is $\sim 8-10 \%$ at ambient temperature. CARS temperature precision improves at higher temperatures, with standard deviation of $60 \mathrm{~K}$ (3\%) at flame temperatures $>2000 \mathrm{~K}$. Spectral features

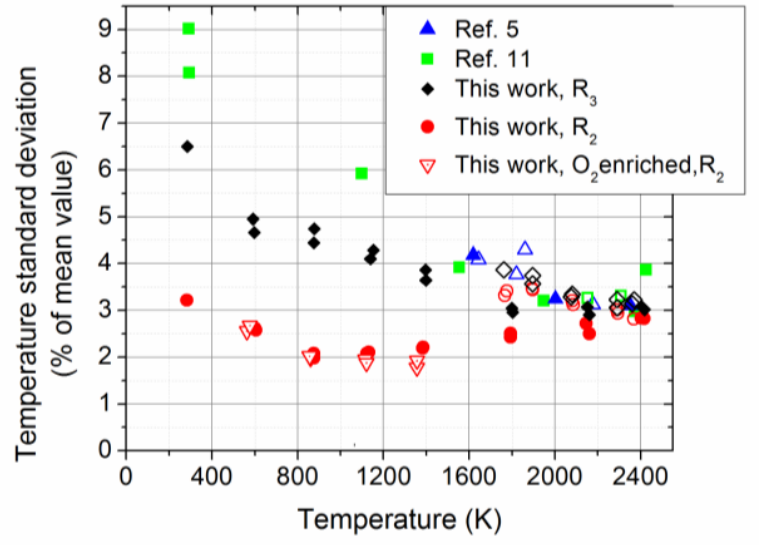

Figure 9. Temperature standard deviation as a function of the measured average temperature. Open symbols are for fuel rich mixtures.

in vibrational CARS spectra strongly depend on temperature; in particular, as temperature increases, $\mathrm{N}_{2}$ and $\mathrm{O}_{2}$ rovibrational structures becomes more marked, and higher order vibrational bands appear above $1000 \mathrm{~K}$, improving the precision at higher temperatures. Still further increasing the temperature, this effect is offset by a reduction of the signal-to-noise ratio. Improved spectral resolution increases the number of structures in low temperature spectra and is therefore helpful in improving the precision at lower temperatures.

Vibrational CARS systems with improved precision (10-15 K) at ambient temperature have been developed. ${ }^{15,41}$, ${ }^{43}$ Such systems are characterized by high spectral resolution, and the use of a single mode pump, and modeless Stokes lasers. A modeless dye laser has smaller mode amplitude fluctuations than a conventional one, therefore reducing the main source of noise in ro-vibrational CARS. Kuehner ${ }^{41}$ reports a standard deviation of 10-15 K in the 90-295 K range. Further improvements were obtained by van Veel ${ }^{15}$ thanks to a simultaneous referencing that takes into account the shot-to-shot fluctuations in the modeless dye laser. Use of a modeless dye laser was considered for this instrument, and attempted in the first preliminary measurements taken in the laboratory-scale free jet, ${ }^{11}$ but results were unsatisfactory.

Figure 9 shows the relative standard deviation computed from 500 single shots measurements in the Hencken burner, and compares it to the results presented in Refs. 5, 11. Green squares are data from Ref. 11, obtained using a modeless dye laser; blue triangles are from Ref. 5 where a conventional dye laser was used. Low signal-to-noise ratio, and narrower bandwidth of the Stokes laser compromised the measurements obtained with the modeless dye laser, and no gain in the instrument precision was observed. Data from Refs. 5 and 11 were analyzed using the algorithm described in Ref. 5, which minimizes the mean square error of the square root of the signal intensity, the residual function $R_{3}$, based on the notation of Ref. 16. This is the same residual minimized by the fitting routine in CARSFT. ${ }^{17}$ The black diamonds are relative standard deviations obtained from the Hencken burner data discussed here, using the fitting algorithm developed by Cutler, ${ }^{16}$ and using $\mathrm{R}_{3}$. Figure 9 shows a $2 \%$ reduction of the relative standard deviation in the 300-1200 $\mathrm{K}$ range caused by the improved spectral resolution achieved with this instrument. At higher temperature, higher order vibrational bands appear and ro-vibrational structures become more marked with the more resolved instrument, but no improvement in precision is observed.

Snelling ${ }^{37}$ observed that CARS temperature precision is improved by fitting to the intensity weighted on a pixel by pixel basis by an estimate of the uncertainty in the intensity. This approach is implemented in the fitting algorithm developed by Cutler ${ }^{16}$ where a reduced $\chi^{2}$ statistic $\left(R_{2}\right)$ residual can be selected in place of $\left.R_{3}\right)$. Red circles are relative standard deviations obtained minimizing the reduced $\chi^{2}$ statistic. As already observed in Ref. 16 a 
significant precision improvement is achieved through the whole temperature range. In particular at $300 \mathrm{~K}$ a temperature standard deviation of only $10 \mathrm{~K}(3.3 \%)$ is obtained. Such a low precision is comparable to the one reported by van Veen using a modeless dye laser, and simultaneous referencing. ${ }^{15}$ The relative standard deviation decreases further by increasing the temperature up to $1200 \mathrm{~K}$, reaching a minimum of $2 \%$. Precision in the 300 $1200 \mathrm{~K}$ range is comparable to what has been reported for pure rotational CARS. Further increasing the temperature, the effect of the reduced signal-to-noise ratio becomes dominant and the relative standard deviation increases, peaking at $3 \%$ at stoichiometric temperature $(2400 \mathrm{~K})$. Note also that at the highest temperature the choice of the residual has very small effect.

For the equivalence ratios above stoichiometric (empty circles), $\mathrm{O}_{2}$ is no longer present, and two or more $\mathrm{H}_{2}$ rotational lines appear. These are isolated lines, and are very sensitive to dye laser mode fluctuations. In particular if two or more $\mathrm{H}_{2}$ rotational lines are present their relative ratio is a function of temperature. The ratio of the two isolated lines strongly depends on the mode noise in the broad-band dye laser and a deleterious effect on temperature precision is expected. To avoid such effects the $S(9)$ line is omitted from the fitting and the $S(5)$ and $\mathrm{S}(6)$ lines are "lumped" together; i.e., in both the theory and the data the two $\mathrm{H}_{2}$ lines are replaced with a region of constant amplitude equal to the mean value over the 2 lines. This lumping removes any influence on the fitted temperature from the $\mathrm{H}_{2}$ spectra and decreases the temperature standard deviation, but the $\mathrm{H}_{2}$ mole fraction standard deviation is slightly increased (for example the temperature and $\mathrm{H}_{2}$ mole fraction standard deviation at $\Phi=3.5$ are 81 $\mathrm{K}$ and 0.03 when the two $\mathrm{H}_{2}$ lines are kept separate, $60 \mathrm{~K}$ and 0.04 when lumped together).

The red open triangles are relative standard deviations obtained for the enriched $\mathrm{O}_{2}$ case. In this case increasing the temperature past $1200 \mathrm{~K}$ further decreases the relative standard deviation with the lowest relative standard deviation $(1.8 \%)$ observed at $1350 \mathrm{~K}$. The reason for the improved precision is the higher concentration of $\mathrm{O}_{2}(\sim 21$ $\%)$ in the enriched case, and therefore the larger signal-to-noise ratio of the $\mathrm{O}_{2}$ spectrum. In the supersonic burner, for the mixing case the center jet is of vitiated air and therefore it has a mole fraction of $\mathrm{O}_{2}$ close to $20 \%$ as in these data. As shown in the insets of Fig. 7, $\mathrm{O}_{2}$ spectra have better spectral resolution than the $\mathrm{N}_{2}$ because the probe beam is the injection seeded Nd:YAG laser. In addition, the Stokes laser center wavelength is chosen to excite the $\mathrm{O}_{2} \mathrm{Q}-$ branch, therefore the mode noise on $\mathrm{O}_{2}$ spectra is lower than on $\mathrm{N}_{2}$. For these reasons measurements in $\mathrm{O}_{2}$ rich mixtures are more precise than in $\mathrm{O}_{2}$ free environments.

The temperature standard deviations reported above also include temporal fluctuations of the flow in the Hencken burner. If the flow had no fluctuations, then the average of the measured fitted single-shot temperatures should coincide with the temperature extracted from the fit of the average of the spectra. This is verified for measurements in ambient air, but not in the flame, where the temperatures from the average spectra are systematically lower than the average of the single shots temperatures. The largest discrepancy is $15 \mathrm{~K}$ and it is observed at stoichiometric conditions. In averaging the spectra, the colder (higher signal) spectra weight more, biasing the temperature to lower values.

Figure 10 shows the standard deviation of the measured mole fractions. The $\mathrm{O}_{2}$ standard deviation stays below $0.6 \%$ of the total mole fraction up to $\Phi=0.5$, then increases up to $1.7 \%$ because of low signal-to-noise ratio. For $\Phi>1$ no $\mathrm{O}_{2}$ is detected, and the single-shot measurements are scattered around a null average value. The $\mathrm{N}_{2}$ mole fraction standard deviation increases from $1.5 \%$ at room temperature to $3.4 \%$ at stoichiometric, and then decreases to $2.5 \%$ at the highest equivalence ratio. The $\mathrm{N}_{2}$ standard deviation as a fraction of the average increases monotonically with the equivalence ratio from $1.7 \%$ to $7 \%$ over this range. The $\mathrm{H}_{2}$ standard deviation increases monotonically with the equivalence ratio, where the standard deviation as fraction of the mean is constant and equal to $10 \%$. The greater precision error in the $\mathrm{H}_{2}$ measurements is largely due to mode noise in the broadband dye laser spectrum, which affects $\mathrm{H}_{2}$ more than $\mathrm{N}_{2}$ or $\mathrm{O}_{2}$ since only two $\mathrm{H}_{2}$ lines are probed, rather than the manifolds of lines for $\mathrm{N}_{2}$ and $\mathrm{O}_{2}$. Mole fraction precision for this instrument is similar to what reported in Refs. 5 and 11 except for $\mathrm{H}_{2}$, which shows a larger standard deviation as a fraction of the mean $(\sim 10 \%$ for this work, $8 \%$ in Ref. $5)$. This is a consequence of moving the $S(5)$ line

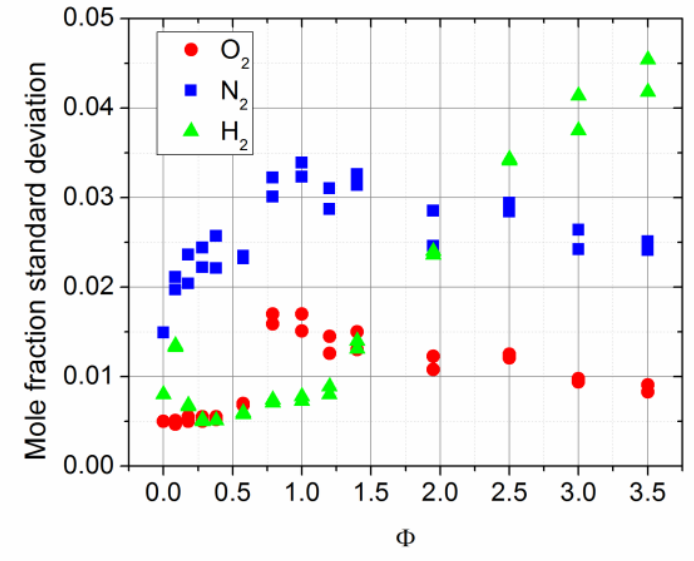

Figure 10. Mole fraction standard deviation as a function of the equivalence ratio $\Phi$. 
toward the tail of the non-resonant spectrum, where the fluctuation (as fraction of the mean) are larger, and of "lumping together" the $S(5)$ and $S(6)$ lines.

\section{Instrument dynamic range}

Study of the axis-symmetric supersonic combusting jet requires an instrument capable of operating in the $300 \mathrm{~K}$ $2400 \mathrm{~K}$ temperature range. Peak CARS signal dependence on temperature is approximated by the relation: ${ }^{44}$

$$
I_{\text {CARS }} \propto T^{-3.5}
$$

The Pixis 100B camera used for this test has a 16 bit dynamic range, therefore a limit of 65535 counts in each pixel of the three bins. The signal is only in the center bin; the upper and lower bins contain only the background. For the Hencken burner dataset here discussed, the $\mathrm{N}_{2}$ peak signal at the highest temperature $(\sim 2400 \mathrm{~K})$ is $\sim 700$ counts above the camera noise level.

Figure 11 plots the peak CARS signal photon counts as a function of temperature; the red circles are observed experimental values, the black curve is obtained from Eq. (2). The figure shows that for temperature below $700 \mathrm{~K}$ the pixels in the proximity of the $\mathrm{N}_{2}$ peak would be saturated. Notice that the scale is logarithmic and for room temperature spectra the number is above one million counts. As mentioned in Section II, we use a half-wave plate and a polarizer to reduce the Nd:YAG energy to prevent detector saturation when the detected signal is too large. The half-waveplate is mounted on a motorized rotation stage controlled by the same Labview code that controls the camera acquisition and the translation stage. During acquisition of a typical dataset, the software moves the translation stage to the desired location; once it has arrived, it collects typically 20 spectra and counts how many have peak intensity above a user-defined threshold (set to 50,000 counts for this work). If $10 \%$ of the spectra are above the threshold, it rotates the waveplate to a

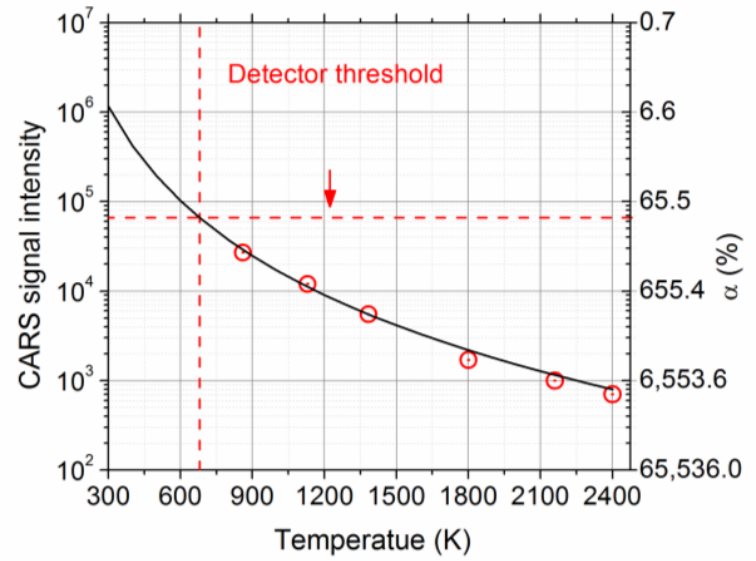

Figure 11. Measured (symbols) and predicted (continuous line) CARS signal intensity in camera counts as a function of the temperature. preset value, reducing the signal intensity, and samples the flow again. The process is iterated until fewer than 2 $(10 \%)$ of the spectra are above the threshold. In previous works, ${ }^{5,9,11}$ the dynamic range issue was solved by adding a neutral density filter in the path of the signal when the signal exceeded the saturation threshold. In those works the process was not automated, and the operator had to verify the threshold level and insert the appropriate neutral density filter. The process described here is completely automated (integrated in the Labview routine that controls the camera and the translation stage), greatly increasing the number of spectra per hour that can be acquired. The approach guarantees that a large percentage ( $90 \%)$ of the shots at a given location does not saturate the camera. Preventing detector saturation in $100 \%$ of the shots, by signal reduction, can be counterproductive in very turbulent region of the flow. In a location where the temperature range is very large, it is preferable to let the coldest $10 \%$ of spectra saturate the camera, rather than lowering the signal below the noise level at the highest temperature. Currently we are unable to analyze spectra with signal that is too low, but we can still extract information from spectra having saturation in some pixels.

In most recent data sets collected at NASA Langley, spectra presenting any saturation were simply thrown away; since colder spectra have larger signal, this approach biases the average measurements toward higher temperature, and artificially reduces the measured range and standard deviation. To avoid the bias, we process the spectra that saturate the camera, but the fitting algorithm is instructed to block the "saturated" pixels when computing the residual function. A numerical test has been performed to evaluate the instrument accuracy when portions of the spectra are not available for fitting. In the numerical test we vary a variable $\alpha$, that is the detector threshold as percentage of the peak value of the processed CARS signal for each test case. In the numerical test $\alpha$ is varied by changing the value of the detector threshold. Every pixel for which the CARS signal is above the threshold, is considered saturated, and is blocked in the residual computation. This is not a perfect reproduction of a spectrum that saturates the camera since we operate on the processed spectrum (with background subtraction and normalization by the non-resonant, but still squared), and we reduce the camera threshold, as opposed to increasing the signal. Also any possible non-linearity of the detector near threshold is neglected. Nonetheless, the simulation provides an indication of the error associated with fitting spectra that saturate the detector. 
Figure 12a shows the percentage difference in the temperature measurements obtained for the unsaturated spectra and for spectra obtained setting the threshold to $\alpha$. Note that decreasing the value of $\alpha$, the portion of spectra blocked from the fitting becomes larger, equivalent to a higher degree of saturation. In practice the threshold value is set (65535 counts), but the CARS peak intensity varies as a function of temperature, composition and beams' alignment. The right axis of Fig. 11 can be used to estimate values of $\alpha$ for given temperatures in the Hencken burner flame. Above $1200 \mathrm{~K}$, the $\mathrm{N}_{2}$ peak is unlikely to be saturated, even when accounting for laser power fluctuations. At $500 \mathrm{~K}$ we expect the threshold to be $\sim 30 \%$ of the peak signal, and only $6 \%$ at room temperature. Based on these considerations, $\alpha$ is varied in the $2 \%-75 \%$ range for this test. For temperature above $1000 \mathrm{~K}$ the error in temperature is below 3\% for any value of $\alpha$ considered. The error is within $1 \%$ when we consider more likely values of $\alpha$ expected in our experiment, such as $2-10 \%$ for room temperature, $10-50 \%$ at $500 \mathrm{~K}$, and $50-100 \%$ above $800 \mathrm{~K}$. Thus, accurate temperatures can be determined from detector saturated spectra taken in fuel-lean mixtures.

Figure $12 \mathrm{~b}$ and $\mathrm{c}$ show errors on the $\mathrm{O}_{2}$ and $\mathrm{N}_{2}$ mole fractions as function of temperature for several values of $\alpha$. In spectra collected with our DP-CARS instrument, in a fuel lean air- $\mathrm{H}_{2}$ flame the $\mathrm{N}_{2}$ signal is always stronger than $\mathrm{O}_{2}$ and therefore more prone to detector saturation. In presence of camera saturation of the $\mathrm{N}_{2}$ spectra, the measured $\mathrm{N}_{2}$ mole fraction is higher and the measured $\mathrm{O}_{2}$ mole fraction lower. The error rapidly increases with decreasing $\alpha$ and with temperature, up to $6 \%$ for the most extreme cases. When considering more likely values of $\alpha$ at each temperature, the error in both mole fractions is within $1 \%$. The test showed that for fuel-lean mixtures, spectra with detector saturation can still produce accurate results, and no significant bias is introduced.


Figure 12. Error in temperature (a), $\mathrm{N}_{2}(\mathrm{~b}), \mathrm{O}_{2}(\mathrm{c})$, and $\mathrm{H}_{2}(\mathrm{~d})$ as function of the temperature and of the ratio between the detector saturation threshold and the CARS signal intensity $(\alpha)$.

For fuel rich mixtures at high temperatures, the $\mathrm{H}_{2} \mathrm{~S}(5)$ line can potentially saturate the detector. Since the $\mathrm{H}_{2}$ lines are not involved in the temperature measurement, there is no significant effect on temperature measurements. In the previous section we have shown that the $S(5)$ and $S(6)$ lines are lumped together in the fitting algorithm. In the presence of an $\mathrm{S}(5)$ line saturating the detector, any information on the line intensity is lost, therefore we exclude the line from the fitting and obtain the mole fraction from the $\mathrm{S}(6)$ line alone. Figure 12d shows that the resulting error is below $1 \%$ in $\mathrm{N}_{2}$ mole fraction, and within $\sim 2 \%$ in the $\mathrm{H}_{2}$. 


\section{DP-CARS Measurements in the Laboratory-Scale Supersonic-Combusting Jet}

In this section we illustrate DP-CARS measurements taken in the jet produced by the laboratory-scale supersonic burner for $\mathrm{M}_{\mathrm{e}}=1.6$ and $\mathrm{M}_{\mathrm{f}}=7$. Results are presented for both mixing $\left(\mathrm{N}_{2}\right.$ co-flow), and combustion $\left(\mathrm{H}_{2}\right.$ co-flow). The CARS probe volume can be approximated by a cylinder of $100 \mu \mathrm{m}$ in diameter and $1.7 \mathrm{~mm}$ long. The probe volume length contains $90 \%$ of the CARS signal, as measured by scanning a $200 \mu \mathrm{m}$ microscope slide cover across the measurement volume. The long measurement volume limits the spatial resolution of the technique, and does not resolve the smallest turbulent structures. But the long volume is required to produce high signal-to-noise ratio while avoiding Raman pumping and Stark effect. ${ }^{12}$ In addition if the temperature in the flow varies along the measurement volume the integrated CARS signal is biased toward lower temperature based on Eq(2). ${ }^{45}$ As shown in Fig. 13, measurement locations reported here are in a Cartesian reference system with origin at the center of the nozzle exit, $z$ axis coincident with the axis of symmetry, $y$ axis along the direction of the Nd:YAG laser beam (and of the CARS probe volume length). Data have been acquired along 5 lines parallel to the $x$ axis and 1, 15, 35, 65 and $100 \mathrm{~mm}$ downstream of the nozzle exit, as shown in Fig. 13. The number of points varies for each line between 27 and 39. Each location along the 65 and $100 \mathrm{~mm}$ line and along the centerline $(x=0)$ is visited 4 times (replications), where points along the 1,15 and $35 \mathrm{~mm}$ lines were visited only once. For each replication, 250 single shot spectra were acquired. A total of $\sim 100,000$ spectra have been collected for each condition.

The data yield (percentage of measured spectra resulting in a successfully fitted temperature and mole fraction) for the mixing case is $97 \%$ and for the combustion case is $95 \%$.

Table 2 shows the measured mean values on the centerline obtained $1 \mathrm{~mm}$ from the nozzle exit at $x=0$ for the mixing case and the combustion case, compared to what is expected at the nozzle exit from previous CFD calculations ${ }^{10}$ (performed only for the combustion case). The difference in the temperature between the mixing and the combustion case is caused by the different heat transfer in the two cases. Temperature is within $4 \%$ of the value computed from the CFD, and the discrepancy is likely due to underestimation of the heat transfer in the numerical model. The instrument measures the same composition for the mixing and the combustion case. This is expected since the mass flow rates are identical; mixing and combustion data were taken in two different days, therefore the results show an excellent reproducibility of both the burner and the

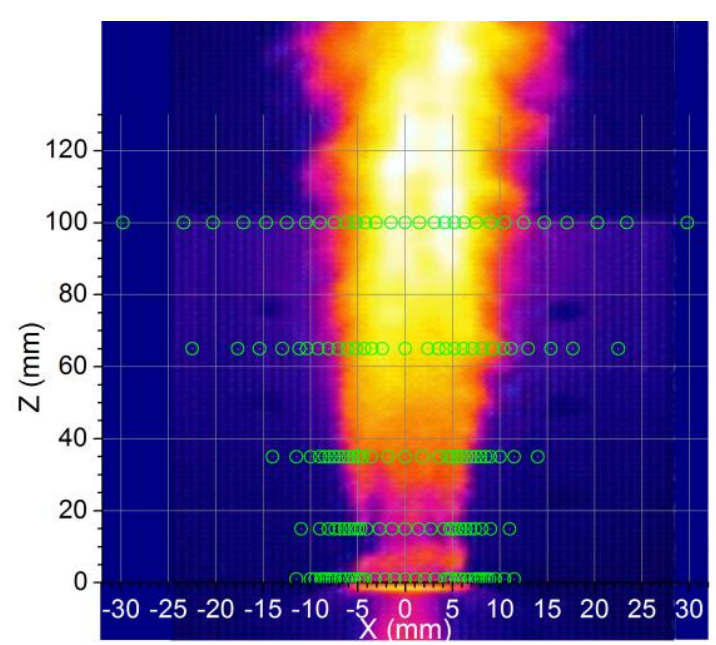

Figure 13. Infra-red image of the supersonic combusting jet. Green circles are the measurement locations.

measurements. The results shown in the table are average of four replications. The average values computed over each replication do not show any trend with time. The standard deviation computed from the 4 average values is 8.4 $\mathrm{K}$ in temperature, 0.001 and 0.003 for $\mathrm{O}_{2}$ and $\mathrm{N}_{2}$ respectively. These values are approximately one order of magnitude lower than the shot-to-shot fluctuations, therefore temporal effects can be neglected. Oxygen mole fraction is on average $8 \%$ higher than predicted. In terms of total mole fraction the $\mathrm{O}_{2}$ is 0.014 higher, and $\mathrm{N}_{2}$ is 0.01 lower than predicted. Measurements in the Hencken burner showed accuracy within 0.005 for $\mathrm{O}_{2}$ and within 0.01 on $\mathrm{N}_{2}$. Errors in the division by the non-resonant spectrum can cause errors up to 0.01 on $\mathrm{O}_{2}$ and 0.025 on $\mathrm{N}_{2}$. Background subtraction is another potential source of error. Errors in the mass flow-rates are also a possible cause for the discrepancy.

Table 2. Comparison of measured and computed centerline mean values.

\begin{tabular}{lccc}
\hline \hline Centerline Mean Values $(z=\mathbf{1 ~ m m})$ & Temperature $(\mathbf{K})$ & $\mathbf{O}_{\mathbf{2}}$ mole fraction & $\mathbf{N}_{\mathbf{2}}$ mole fraction \\
\hline Measured (Mixing) & 1373 & 0.20 & 0.50 \\
Measured (Combustion) & 1395 & 0.20 & 0.50 \\
Computed (Combustion, [10]) & 1458 & 0.186 & 0.51 \\
\hline Difference \% & $4.3 \%$ & $7.5 \%$ & $2 \%$ \\
\hline \hline
\end{tabular}



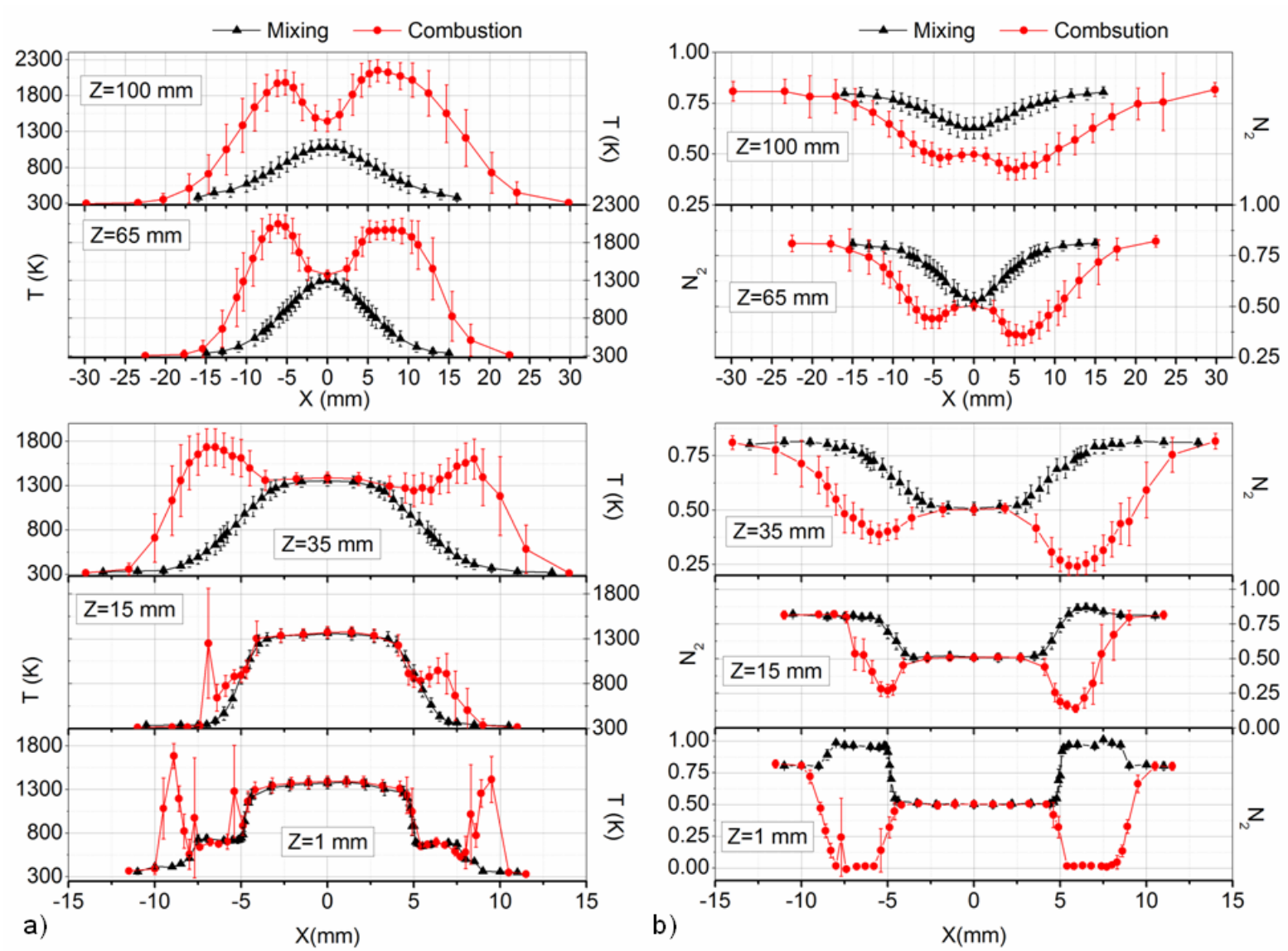

Figure 14. a) Measured temperature and b) $\mathbf{N}_{2}$ profiles at different heights for mixing (in black) and combustion (in red). Error bars indicate $+/-1$ standard deviation of the measurement.

Figures 14 and 15 show the DP-CARS average measurements of temperature and mole fractions, as a function of $x$ at different heights, $z$. The black symbols are for the mixing case, the red for the combustion case. The average at each location is computed over the total number of replications and repetitions. The error bars in the figures represent \pm one standard deviation, and include fluctuations due to both the instrument precision and the turbulence in the flow. The two contributions can be isolated, based on the knowledge of the instrument precision as function of temperature and composition.

The lowest panels in Figs. 14 and 15 represent data taken $1 \mathrm{~mm}$ from the nozzle exit. This is the lowest height probed. The mixing data (black symbols) show a uniform profile in the jet core up to $\pm 4 \mathrm{~mm}$ from the nozzle center. Between $x= \pm 4$ and $\pm 5 \mathrm{~mm}$ there is a mixing layer between the center supersonic jet of vitiated air and the $\mathrm{N}_{2}$ coflow. This region is characterized by a rapid drop in temperature from 1300 to $720 \mathrm{~K}$, a reduction in the $\mathrm{O}_{2}$ concentration and an increase in $\mathrm{N}_{2}$. Relatively large fluctuations are present in these regions: measured standard deviations $4.9 \mathrm{~mm}$ from the center are $110 \mathrm{~K}$ in temperature, 0.10 and 0.04 for $\mathrm{N}_{2}$ and $\mathrm{O}_{2}$ mole fractions, respectively. (At the centerline standard deviations are $62 \mathrm{~K}, 0.03$ and 0.01, respectively.) Between 5 and $7.5 \mathrm{~mm}$ from the centerline the temperature stays constant, and the concentration changes toward a pure $\mathrm{N}_{2}$ co-flow. Shot-toshot fluctuations in this region are lower than in the center jet. Measurements taken $7.5 \mathrm{~mm}$ from the nozzle center are in the shear layer between the $\mathrm{N}_{2}$ co-flow and the ambient air. Past this location the temperature quickly drops, entering the ambient air region. Regions past $11.5 \mathrm{~mm}$ from the axis were not measured, and a location where the ambient air is completely unaffected by the flow is not reached. Moving downstream, $15 \mathrm{~mm}$ from the exit, the center jet remains unaffected by the co-flow but the temperature profile no longer shows the plateau in the co-flow region. The large amount of $\mathrm{O}_{2}$ present indicates that a significant amount of air is entrained by the co-flow. Mole fraction measurements are asymmetric with higher $\mathrm{N}_{2}$ and lower $\mathrm{O}_{2}$ mole fraction for positive $x$. The asymmetry is likely to be caused by small errors in centering the nozzle with respect to the annulus, thereby increasing the co-flow nozzle exit area on one side, and reducing it on the other. This causes non-symmetric mass flow-rates in the co-flow, higher for positive $x$. At $z=35 \mathrm{~mm}$ above the nozzle, mixing between the supersonic center jet and the co-flow 
becomes significant but the inner region of the jet ( $\pm 3 \mathrm{~mm}$ from the axis) is still unaltered. The concentration asymmetry observed at $15 \mathrm{~mm}$ propagates downstream, but it is much weaker because of the mixing. At $15 \mathrm{~mm}$ a difference up to 0.06 in $\mathrm{O}_{2}$ mole fraction is observed between two symmetrical points, where at $35 \mathrm{~mm}$ the maximum difference recorded is 0.02 . Further downstream, 65 and $100 \mathrm{~mm}$ from the base, mixing involves the entire center jet, and the asymmetry is no longer significant. At these heights each location was visited 4 times, for a total of $\sim 1000$ spectra collected at each location, allowing for better estimates of the second order statistics.
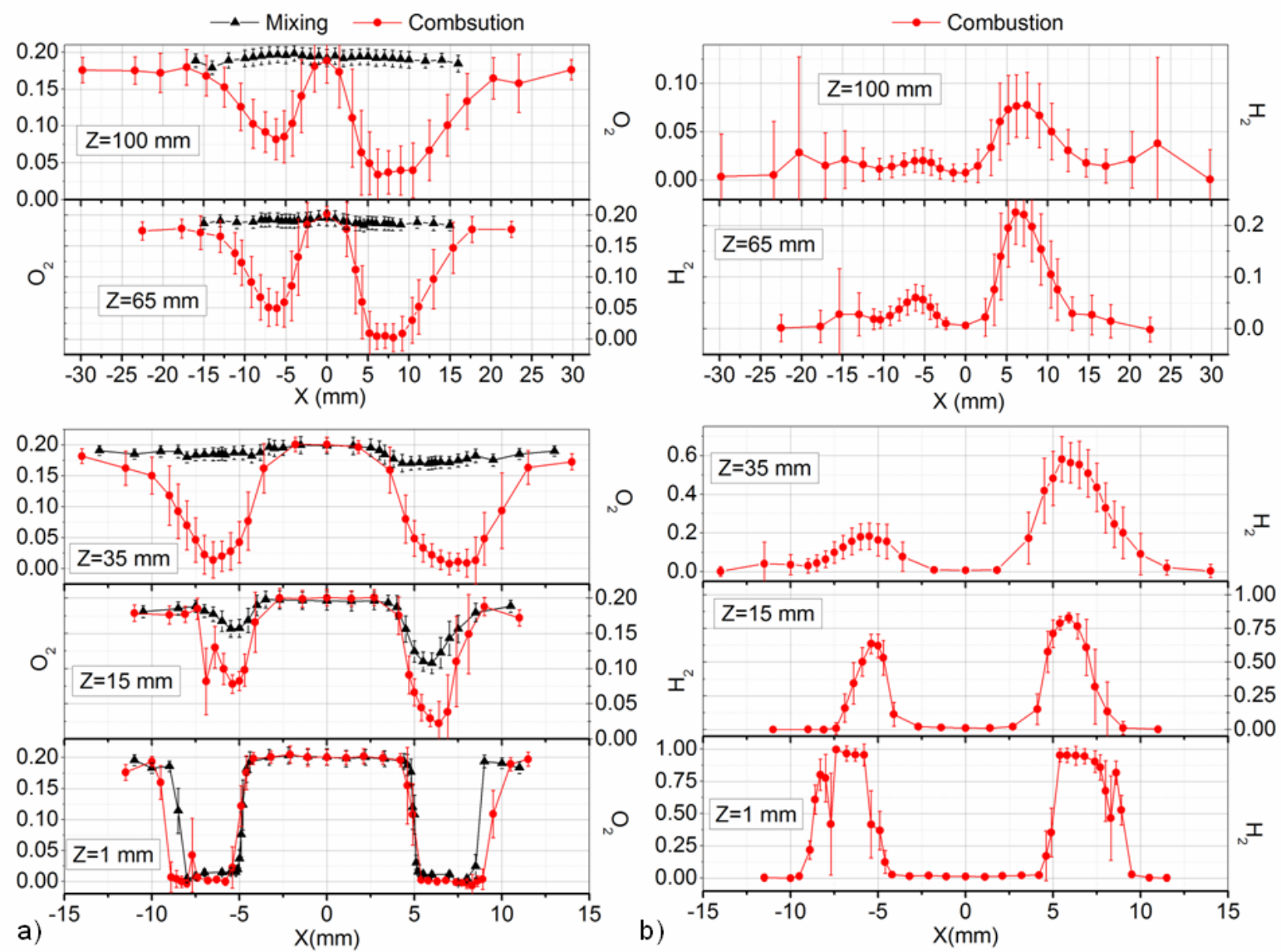

Figure 15. a) Measured $\mathrm{O}_{2}$ and b) $\mathrm{H}_{2}$ profiles at different heights for mixing (in black) and combustion (in red). Error bars indicate +/- 1 standard deviation of the measurement.

The combustion case presents a much more complex profile. One $\mathrm{mm}$ above the nozzle the mixing and combustion profiles overlaps in the range of $x= \pm 4 \mathrm{~mm}$. Beyond $4 \mathrm{~mm}$ from the centerline the two cases begin to differentiate because of the different co-flow composition. Temperature profiles are very similar to what is observed for the mixing for $x$ between \pm 4 and $\pm 7.4 \mathrm{~mm}$, with a mixing layer characterized by large fluctuations followed by a less turbulent flow corresponding to the $\mathrm{H}_{2}$ co-flow. Measurements were collected also in the pure $\mathrm{H}_{2}$ co-flow region. Temperature was high enough so that two $\mathrm{H}_{2}$ lines, $\mathrm{S}(5)$ and $\mathrm{S}(6)$, were detected. When possible we prefer to extract the temperature from the $\mathrm{N}_{2}$ spectra, but for this region we obtain the temperature measurement by the two $\mathrm{H}_{2}$ lines. No data are available for the accuracy and precision of these measurements. The average temperature in the co-flow is $\sim 670 \mathrm{~K}$, and the standard deviation as low as $35 \mathrm{~K}$ - similar to what is measured in the $\mathrm{N}_{2}$ co-flow. The measured $\mathrm{H}_{2}$ concentration in the pure $\mathrm{H}_{2}$ co-flow is between 0.95 and 1 , and the instrument correctly measures a null mole fraction of $\mathrm{N}_{2}$ and $\mathrm{O}_{2}$. Past $7.4 \mathrm{~mm}$ from the center, mixing between the co-flow and ambient air occurs, and temperature is sufficiently high to allow self-ignition. The annular combustion region is $\sim 2 \mathrm{~mm}$ thick and contains 6 measurement locations, but it appears un-resolved. The temperature plots shows local peaks for $x=-5.4$, 7.7 and $8.3 \mathrm{~mm}$. Measurements at these locations present very large fluctuations, up to $690 \mathrm{~K}$ in temperature. Examining the evolution with time of the single shots measurements, we noticed that the first shots were from high temperature fuel rich mixtures, and the rest by much colder spectra. Movement of the translation stage during the data acquisition could explain this behavior, but the symmetry in the location of these regions makes this explanation unlikely. Another possibility is that sparks produced by the focused laser beams ignites the mixture, but 
the flame cannot self-sustain. Unfortunately no replications are available for these locations. At $z=15$ $\mathrm{mm}$ from the nozzle exit, the temperature profile is significantly different. The main feature is a strong asymmetry with respect to the axis. For positive $x$ the combustion region is closer to the center jet, and is characterized by a lower-temperature, fuel-rich flame. For negative $x$ the temperature in the co-flow region is lower, and both $\mathrm{H}_{2}$ and $\mathrm{O}_{2}$ are still present. Single-shot spectra show simultaneous presence of $\mathrm{O}_{2}$ and $\mathrm{H}_{2}$, therefore the combustion is not sustained in this region. For $x=-6.9$ the average temperature is $1270 \mathrm{~K}$, but the time history of the single shots temperatures shows high temperatures for the first 4 seconds of acquisition and lower thereafter. A spark caused by the lasers itself could have ignited the flow, but conditions were inadequate to sustain the combustion, as suspected for those other anomalous location in the lower plane $(x=-$ 5.4, -7.7, and $8.3 \mathrm{~mm}$ ). Flow rates of $\mathrm{H}_{2}$ appear to be higher (higher mole fraction) for positive $x$, which is consistent with observations in the mixing case. The asymmetry in the flow-rates causes different compositions for positive and negative values of $x$, which allow sustained combustion on one side of the centerline but not the other. Note that the asymmetry is also visible in the IR image of Fig. 13 . At $z=35 \mathrm{~mm}$, combustion between the $\mathrm{H}_{2}$ co-flow and the ambient air occurs for both positive and negative values of $x$. The temperature and composition distribution is asymmetric, with a richer and lower temperature flame for positive $x$. Mixing with the center jet is present at this location, but there is no evidence of supersonic combustion. Further downstream, $65 \mathrm{~mm}$ from the nozzle exit combustion between the $\mathrm{O}_{2}$ in the supersonic jet and the $\mathrm{H}_{2}$ in the co-flow has occurred. $100 \mathrm{~mm}$ from the burner base combustion still occurs for positive $x$, but $\mathrm{H}_{2}$ is completely depleted for negative $x$.

In addition to average and standard deviations, covariances and histograms at each location have been obtained to provide additional information to the CFD modelers. Figure 16 shows histograms of temperature measurements at the nozzle exit $(x=0$ and $z=1 \mathrm{~mm}$, in the lower panel) and in a combustion region downstream $(x=14.7$ and $z=100 \mathrm{~mm}$, in the upper panel). The temperature histogram in the lower panel has a Gaussian distribution (black curve, $\mathrm{R}^{2}=0.99$ ). The temperature histogram obtained in the combustion region downstream has a much larger standard deviation $(285 \mathrm{~K})$ than is observed at the nozzle exit $(62 \mathrm{~K})$, and does not follow a Gaussian distribution (dotted curve in figure, $\mathrm{R}^{2}=0.77$ ). Instead has a bimodal distribution (black continuous line, $\mathrm{R}^{2}=0.97$ ), given by the sum of a Gaussian centered at $1500 \mathrm{~K}$ (blue curve) and another Gaussian centered at $1986 \mathrm{~K}$ (green curve). The bimodal distribution indicates fluctuations of the flame front. The temperature histogram in the combustion region shows temperature ranging from 400 to $2400 \mathrm{~K}$ in a single location. Removing spectra that saturate the detector from the analysis, as in Refs. 9,11 , would have neglected those lower temperature shots introducing errors in the average and in the standard deviation estimates.



Figure 17. Histograms of temperature at $x=0, z=1$ (lower panel) and at $x=14.7, z=100$ (upper panel). The black continuous lines are fit to a Gaussian (lower panel) and to the sum of 2 Gaussian (in blue and green).

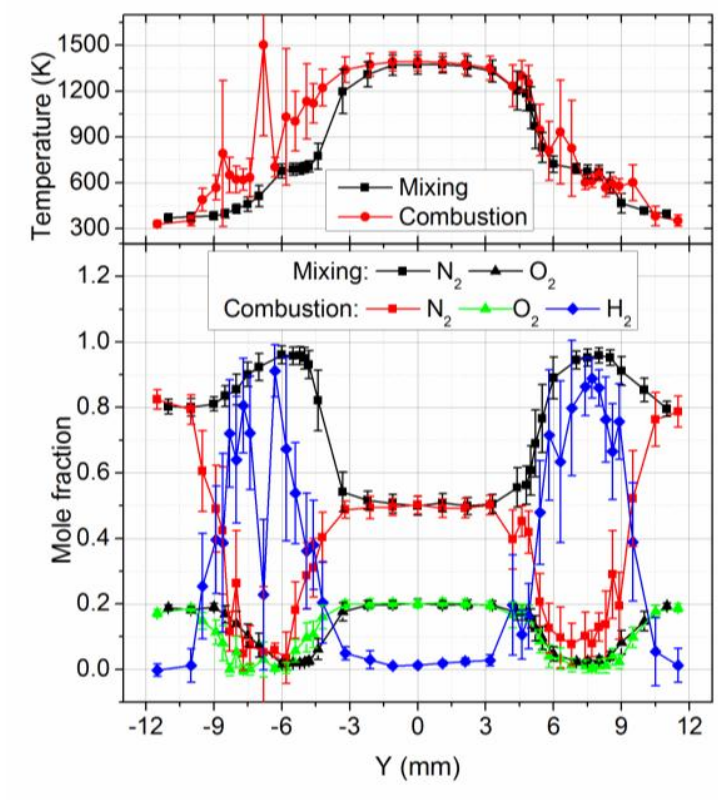

Figure 16. Temperature (top panel) and mole fraction measurements along the $y$ direction, $1 \mathrm{~mm}$ above the nozzle exit. 
In order to compare experimental and computational results, it is necessary to know the exact location at which the data have been collected. In section II the procedure to determine the location of the nozzle center in the reference frame of the translation stage is described. The algorithm relies on quickly analyzing just a few samples of unprocessed spectra. This can lead to errors of $1 \mathrm{~mm}$ or more. The profiles of temperature and mole fractions obtained near the nozzle exit, combined with the symmetry of the center jet allow for a more precise determination of the nozzle center. To obtain an improved estimate of the origin $(x=0, y=0)$ location with respect to the actual centerline, we fit temperature profiles in the core of the jet (over a region of $\pm 3.3 \mathrm{~mm}$ for the mixing case, $\pm 4.6 \mathrm{~mm}$ for the combustion case) with a quadratic function; the vertex of the fitted parabola provides the actual location of the centerline. Values determined along $x$ are $-0.016 \mathrm{~mm}$ for the mixing, and -0.05 for the combustion, therefore they are negligible. Figure 17 summarizes measurements obtained $1 \mathrm{~mm}$ from the exit along the $y$ direction. It is evident that for the mixing case the origin is not along the axis of symmetry. From the quadratic fitting we obtain that the centerline is $0.55 \mathrm{~mm}$ from the origin of the reference system for the mixing data, and $0.06 \mathrm{~mm}$ for the combustion data. This approach allows accurate determination of the location of the measurement points.

\section{Conclusion}

A dual-pump CARS system for measurements of temperature and absolute mole fractions of $\mathrm{N}_{2}, \mathrm{O}_{2}$ and $\mathrm{H}_{2}$ in a supersonic combusting free jet has been developed. The instrument and the data analysis approach are described with emphasis on procedures developed to acquire data in a supersonic, turbulent, high-temperature, chemicallyreacting environment. Several issues affecting the quality of the measurements in previous attempts have been resolved. Results from validation measurements in a Hencken burner are discussed and the instrument accuracy and precision estimated. Calibration of the optical diffusion coefficient and the third order non-resonant susceptibility reduced systematic errors. The accuracy of the mole fractions measured for temperatures above $600 \mathrm{~K}$ is $0.5,1.5$ and $1 \%$ of the total composition for $\mathrm{O}_{2}, \mathrm{~N}_{2}$ and $\mathrm{H}_{2}$, respectively. Uncertainty on the non-resonant spectrum is responsible for additional errors up to $5 \%$ of the measured mole fraction, and up to $2 \%$ of the measured temperature. The precision of the temperature measurements, determined from the standard deviation of the measured temperature over 500 acquisitions, is function of temperature and composition, and lies between 1.8 and $3.3 \%$. Absolute mole fraction precisions are between 0.5 and $1.8 \%$ for $\mathrm{O}_{2}, 1.5$ and $3.4 \%$ for $\mathrm{N}_{2}$, where these percentages are given as a fraction of the total composition. The precision of $\mathrm{H}_{2}$ mole fraction measurements is $\sim 10 \%$ of the measured value.

Results from a numerical study show that accurate temperature and species mole fraction measurements are possible when a portion of the spectra saturates the camera. The error is within $1 \%$ for the degree of detector saturation expected in the supersonic combusting jet. This approach is highly preferable to the removal of the saturated spectra because does not introduce significant bias, and extend the range of temperature that the instrument is capable of measuring.

The instrument has been used to collect data in a laboratory-scale supersonic-combusting jet for several values of exit and flight Mach number, though only one pair of cases (mixing and combustion) was reported here. Approximately 200,000 spectra have been analyzed. The signal-to-noise ratio was sufficiently high at all the locations tested, and a data yield $>95 \%$ was achieved. Averages, standard deviations, co-variances and histograms were obtained at each location probed. The averages profiles show that the combustion begins at the interface between the $\mathrm{H}_{2}$ annular co-flow and the ambient air. At $z=65 \mathrm{~mm}$ combustion between the center jet of vitiated air and the co-flow has occurred. The average profiles show an asymmetry in the flow, caused by a non-uniform exit area in the co-flow nozzle.

Temperature standard deviations are consistently larger (2x or more) than what is measured in laminar flames, (for example at the nozzle exit the measured standard deviation is $62 \mathrm{~K}$, where in a laminar flame at similar temperature and composition is $26 \mathrm{~K}$ ). Therefore the instrument is capable of estimating temperature fluctuations in this flow. Mole fraction standard deviations at the nozzle exit are of the same order of magnitude of what is observed in a laminar flame, and are therefore not resolved by the technique. At the flame front or $35 \mathrm{~mm}$ above the nozzle exit the mole fraction fluctuations become larger and can be resolved. The remainder of the data will be presented in future publications. In particular, the effect on mixing and combustion of different exit and flight Mach numbers will be discussed. The purpose of this test campaign was to provide data to CFD modelers to improve the computational models for supersonic mixing and combustion. Unfortunately the asymmetry in the flow makes comparison with CFD results more challenging. The co-flow nozzle, responsible for the asymmetry has been redesigned and further DP-CARS test in this flow are planned. 


\section{Acknowledgments}

This work was supported by the NASA Fundamental Aeronautics Program, Hypersonics Project and the NASA AFOSR-sponsored National Center for Hypersonic Combined Cycle Propulsion. The authors would like to thank Lloyd G. Wilson and Sarah A. Tedder for assistance in performing these experiments.

\section{References}

1. Anderson, T. J., and Eckbreth, A. C. "Simultaneous Coherent Anti-Stokes Raman Spectroscopy Measurements in Hydrogen Fueled Supersonic Combustion," Journal of Propulsion and Power Vol. 8, No. 1, 1992, pp. 7-15,

2. $\quad$ Yang, S. R., Zhao, J. R., Sung, C. J., and Yu, G. "Multiplex CARS measurements in supersonic H2/air combustion," Applied Physics B: Lasers and Optics Vol. 68, No. 2, 1999, pp. 257-265, doi: 10.1007/s003400050615

3. Cutler, A. D., Danehy, P. M., Springer, R. R., O'Byrne, S., Capriotti, D. P., and DeLoach, R. "Coherent Anti-Stokes Raman Spectroscopic Termomethry in a Supersonic Combustor," AIAA Journal Vol. 41, No. 12, 2003,

4. Lucht, R. P. "Three-laser coherent anti-Stokes Raman scattering measurements of two species," Opt. Lett. Vol. 12, No. 2, 1987, pp. 78-80,

5. O'Byrne, S., Danehy, P. M., Tedder, S. A., and Cutler, A. D. "Dual Pump Coherent Anti-Stokes Raman Scattering Measurements in Supesonic Combustor," AIAA Journal Vol. 45, No. 4, 2007, pp. 922-933,

6. Cutler, A. D., Magnotti, G., Baurle, R., Bivolaru, D., Tedder, S. A., Danehy, P. M., Weikl, M., Beryrau, F., and Seeger, T. "Development of Supersonic Combustion Experiments for CFD Model development," AIAA 2008-0978, 45th AIAA Aerospace Sciences Meeting, Reno, NV, 2008

7. Cutler, A. D., Magnotti, G., Capriotti, D. P., and Mills, C. T. "Supersonic Combusting Jet Experiments for Code Development and Validation," Paper-1150, 55th JANNAF Propulsion Meeting, Boston, MA, 2008

8. Baurle, R., and Edwards, J. R. "Hybrid Reynolds-Average/ Large Edddy Simulationsnof a Coaxial Supersonic Free Jet Experiment," AIAA-2009-0524, 47th AIAA Aerospace Sciences Meeting, Orlando, FL., 2009

9. Tedder, S. A., Danehy, P. M., Magnotti, G., and Cutler, A. D. "CARS Temperature Measurements in a CombustorHeated Mach 1.6 Jet," AIAA-2009-0524 47 ${ }^{\text {th }}$ Aerospace Sciences Meeting, Orlando, FL, 2009

10. Magnotti, G., and Cutler, A. D. "An Experimental and Numerical Study of a Supersonic Burner for CFD Development," AIAA 2008-4686 44th AIAA/ASME/SAE/ASEE Joint Propulsion Conference \& Exhibit, Hartford, CT, 2008

11. Tedder, S. A., Bivolaru, D., and Danehy, P. M. "Characterization of a Combined CARS and Interferometric Rayleigh Scattering System," AIAA 2007-871 45th AIAA Aerospace Science Meeting, Reno, 2007

12. Magnotti, G., Cutler, A. D., Herring, G. C., Tedder, S. A., and Danehy, P. M. "Saturation and Stark Broadening Effects in Dual-Pump CARS of N2, O2 and H2," Journal of Raman Specroscopy, 2012,

13. Magnotti, G., Cutler, A. D., and Danehy, P. M. "Beam Shaping for CARS Measurements in Turbulent Environments," AIAA-2010-1400 48 ${ }^{\text {th }}$ AIAA Aerospace Sciences Meeting, Orlando, FL, 2010

14. Bivolaru, D., and Herring, G. C. "Focal Plane Imaging of Crossed Beams in Non-linearOptics Experiments," Review of Scientific Instruments Vol. 78, No. 5, 2007,

15. van Veen, E. H., and Roekaerts, D. "Thermometry for turbulent flames by coherent anti-Stokes Raman spectroscopy with simultaneous referencing to the modeless excitation profile," Appl. Opt. Vol. 44, No. 32, 2005, pp. 6995-7004,

16. Cutler, A. D., and Magnotti, G. "CARS Spectral Fitting with Multiple Resonant Species Using Sparse Libraries," Journal of Raman Specroscopy Vol. in press, 2010,

17. Palmer, R. E. "The CARSFT Computer Code for Calculating Coherent Anti-Stokes Raman Spectra: User and Programmer Information." Vol. SAND89-8206, SANDIA National Labs, Livermore, CA, 1989.

18. Hancock, R. D., Schauer, F. R., Lucht, R. P., and Farrow, R. L. "Dual-pump coherent anti-Stokes Raman scattering measurements of nitrogen and oxygen in a laminar jet diffusion flame," Appl. Opt. Vol. 36, No. 15, 1997, pp. 32173226,

19. Danehy, P. M., O'Byrne, S., and Cutler, A. D. "Coherent Anti-Stokes Raman Scattering (CARS) as a probe for Supersonic Hydrogen-Fuel/Air Mixing," Proceedings of the JANNAF APS/CS/PSHS/MSS Joint Meeting, 2003

20. Tipping, R. H., and Bouanich, J. P. "On the use of Herman-Wallis factors for diatomic molecules," Journal of Quantitative Spectroscopy and Radiative Transfer Vol. 71, No. 1, 2001, pp. 99-103, doi: 10.1016/s00224073(01)00014-0

21. James, T. "Line Intensities in the Raman Effect of 1? Diatomic Molecules," Journal of Chemical Physics Vol. 31, No. 1, 1959, p. 130,

22. Marrocco, M., Magnotti, G., and Cutler, A. D. "Herman-Wallis corrections in dual-pump CARS intensities for combustion temperature and species," Journal of Raman Spectroscopy Vol. in press, 2012,

23. Hancock, R. D., Bertagnolli, K. E., and Lucht, R. P. "Nitrogen and hydrogen CARS temperature measurements in a hydrogen/air flame using a near-adiabatic flat-flame burner," Combustion and Flame Vol. 109, No. 3, 1997, pp. 323331, doi: 10.1016/s0010-2180(96)00191-5 
24. Lundeen, T., Hou, S.-Y., and Nibler, J. W. "Nonresonant third order susceptibilities for various gases," Journal of Chemical Physics Vol. 79, No. 12, 1983, p. 6301,

25. Hall, R. J., and Greenhalgh, D. A. "Application of the rotational diffusion model to gaseous N2 CARS spectra," Optics Communications Vol. 40, No. 6, 1982, pp. 417-420, doi: 10.1016/0030-4018(82)90044-x

26. Kojima, J., and Nguyen, Q.-V. "Quantitative analysis of spectral interference of spontaneous Raman scattering in highpressure fuel-rich H2-air combustion," Journal of Quantitative Spectroscopy and Radiative Transfer Vol. 94, No. 3-4, 2005, pp. 439-466, doi: 10.1016/j.jqsrt.2004.10.004

27. Varghese, P. L., and Hanson, R. K. "Collisional narrowing effects on spectral line shapes measured at high resolution," Appl. Opt. Vol. 23, No. 14, 1984, pp. 2376-2385,

28. Bischel, W. K., and Dyer, M. J. "Temperature dependence of the Raman linewidth and line shift for the Q(1) and Q(0) transitions in normal and para-H_\{2\}," Physical Review A Vol. 33, No. 5, 1986, p. 3113,

29. Duggan, P., Sinclair, P. M., Le Flohic, M. P., Forsman, J. W., Berman, R., May, A. D., and Drummond, J. R. "Testing the validity of the optical diffusion coefficient: Line-shape measurements of CO perturbed by N_\{2\}," Physical Review A Vol. 48, No. 3, 1993, p. 2077,

30. La, S., and Harris, L. E. "Relative value of the third-order nonresonant susceptibility ofwater," Appl. Opt. Vol. 25, No. 23, 1986, pp. 4501-4503,

31. Rosasco, G. J., and Hurst, W. S. "Dispersion of the electronic contribution to the third-order nonlinear susceptibility of H2," J. Opt. Soc. Am. B Vol. 3, No. 10, 1986, pp. 1251-1256,

32. Farrow, R. L., Lucht, R. P., and Rahn, L. A. "Measurements of the nonresonant third-order susceptibilities of gases using coherent anti-Stokes Raman spectroscopy," J. Opt. Soc. Am. B Vol. 4, No. 8, 1987, pp. 1241-1246,

33. Shelton, D. P., and Rice, J. E. "Measurements and calculations of the hyperpolarizabilities of atoms and small molecules in the gas phase," Chemical Reviews Vol. 94, No. 1, 1994, pp. 3-29, doi: 10.1021/cr00025a001

34. Hahn, J. W., and Lee, E. S. "Measurement of nonresonant third-order susceptibilities of various gases by the nonlinear interferometric technique," J. Opt. Soc. Am. B Vol. 12, No. 6, 1995, pp. 1021-1027,

35. Hauchecorne, G., Kerherve, F., and Mayer, G. "Mesure des interactions entre ondes lumineuses dans diverses substances," Journal de Physique Vol. 32, 1971, p. 47,

36. Pealat, M., Bouchardy, P., Lefebvre, M., and Taran, J. P. "Precision of multiplex CARS temperature measurements," Appl. Opt. Vol. 24, No. 7, 1985, pp. 1012-1022,

37. Snelling, D. R., Smallwood, G. J., Sawchuk, R. A., and Parameswaran, T. "Precision of multiplex CARS temperatures using both single-mode and multimode pump lasers," Appl. Opt. Vol. 26, No. 1, 1987, pp. 99-110,

38. Kröll, S., Aldén, M., Bengtsson, P. E., and Löfström, C. "An evaluation of precision and systematic errors in vibrational CARS thermometry," Applied Physics B: Lasers and Optics Vol. 49, No. 5, 1989, pp. 445-453, doi: $10.1007 / \mathrm{bf00325348}$

39. Prucker, S., Meier, W., and Stricker, W. "A flat flame burner as calibration source for combustion research: Temperatures and species concentrations of premixed H2/air flames," Review of Scientific Instruments Vol. 65, No. 9, 1994, p. 2908,

40. Seeger, T., and Leipertz, A. "Experimental comparison ofsingle-shot broadband vibrationaland dual-broadband pure rotationalcoherent anti-Stokes Raman scattering in hot air," Appl. Opt. Vol. 35, No. 15, 1996, pp. 2665-2671,

41. Kuehner, J. P., Woodmansee, M. A., Lucht, R. P., and Dutton, J. C. "High-Resolution Broadband N2 Coherent AntiStokes Raman Spectroscopy: Comparison of Measurements for Conventional And Modeless Broadband Dye Lasers," Appl. Opt. Vol. 42, No. 33, 2003, pp. 6757-6767,

42. Hartung, G., Walewski, J. W., Hult, J. F., and Kaminski, C. F. "A flat flame burner for the calibration of laser thermometry techniques," Measurement Science \& Technology Vol. 17, 2005, p. 2485,

43. Hahn, J. W., Park, C. W., and Park, S. N. "Broadband coherent anti-Stokes Raman spectroscopy with a modeless dye laser," Appl. Opt. Vol. 36, No. 27, 1997, pp. 6722-6728,

44. Bultitude, K. M., Danehy, P. M., Fraval, E., Fox, J. S., and Houwing, A. F. P. "Broadband coherent anti-Stokes Raman spectroscopy (BB-CARS) in flames and hypersonic flows," 2nd Australian Conference on Laser Diagnostics in Fluid Mechanics and Combustion, Monash University, Melbourne Australia 1999

45. Parameswaran, T., and Snelling, D. R. "Estimation of spatial averaging of temperatures from coherent anti-Stokes Raman spectroscopy," Appl. Opt. Vol. 35, No. 27, 1996, pp. 5461-5464, 